\title{
Results from a Systematic Survey of X-Ray Emission from Hydrogen-poor Superluminous SNe
}

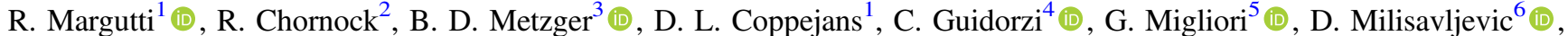

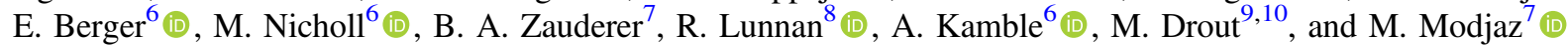 \\ ${ }^{1}$ Center for Interdisciplinary Exploration and Research in Astrophysics (CIERA) and Department of Physics and Astronomy, Northwestern University, Evanston, \\ IL 60208, USA \\ ${ }^{2}$ Astrophysical Institute, Department of Physics and Astronomy, 251B Clippinger Lab, Ohio University, Athens, OH 45701, USA \\ ${ }^{3}$ Columbia Astrophysics Laboratory, Columbia University, Pupin Hall, New York, NY 10027, USA \\ ${ }^{4}$ Department of Physics and Earth Science, University of Ferrara, via Saragat 1, I-44122, Ferrara, Italy \\ ${ }^{5}$ Laboratoire AIM (CEA/IRFU—CNRS/INSU-Universite Paris Diderot), CEA DSM/SAp, F-91191 Gif-sur-Yvette, France \\ ${ }^{6}$ Harvard-Smithsonian Center for Astrophysics, 60 Garden Street, Cambridge, MA 02138, USA \\ ${ }^{7}$ Center for Cosmology and Particle Physics, New York University, 4 Washington Place, New York, NY 10003, USA \\ ${ }^{8}$ Department of Astronomy, California Institute of Technology, 1200 East California Boulevard, Pasadena, CA 91125, USA \\ ${ }^{9}$ Carnegie Observatories, 813 Santa Barbara Street, Pasadena, CA 91101, USA \\ Received 2017 April 18; revised 2018 June 23; accepted 2018 June 27; published 2018 August 29
}

\begin{abstract}
We present the results from a sensitive X-ray survey of 26 nearby hydrogen-poor superluminous supernovae (SLSNe-I) with Swift, Chandra, and XMM. This data set constrains the SLSN evolution from a few days until $\sim 2000$ days after explosion, reaching a luminosity limit $L_{x} \sim 10^{40} \mathrm{erg} \mathrm{s}^{-1}$ and revealing the presence of significant X-ray emission possibly associated with PTF 12dam. No SLSN-I is detected above $L_{x} \sim 10^{41} \mathrm{erg} \mathrm{s}^{-1}$, suggesting that the luminous X-ray emission $L_{x} \sim 10^{45} \mathrm{erg} \mathrm{s}^{-1}$ associated with SCP 60F6 is not common among SLSNe-I. We constrain the presence of off-axis gamma-ray burst (GRB) jets, ionization breakouts from magnetar engines and the density in the sub-parsec environments of SLSNe-I through inverse Compton emission. The deepest limits rule out the weakest uncollimated GRB outflows, suggesting that if the similarity of SLSNe-I with GRB/SNe extends to their fastest ejecta, then SLSNe-I are either powered by energetic jets pointed far away from our line of sight $\left(\theta>30^{\circ}\right)$, or harbor failed jets that do not successfully break through the stellar envelope. Furthermore, if a magnetar central engine is responsible for the exceptional luminosity of SLSNe-I, our X-ray analysis favors large magnetic fields $B>2 \times 10^{14} \mathrm{G}$ and ejecta masses $M_{\mathrm{ej}}>3 M_{\odot}$, in agreement with optical/UV studies. Finally, we constrain the pre-explosion mass-loss rate of stellar progenitors of SLSNe-I. For PTF 12dam we infer $\dot{M}<2 \times 10^{-5} M_{\odot} \mathrm{yr}^{-1}$, suggesting that the SN shock interaction with an extended circumstellar medium is unlikely to supply the main source of energy powering the optical transient and that some SLSN-I progenitors end their lives as compact stars surrounded by a low-density medium similar to long GRBs and type $\mathrm{Ib} / \mathrm{c} \mathrm{SNe}$.
\end{abstract}

Key words: stars: magnetars - stars: mass-loss - supernovae: general - X-rays: general

Supporting material: machine-readable table

\section{Introduction}

Superluminous supernovae (SLSNe) are among the most luminous known stellar explosions in the universe. Only recently recognized as a class, in 2009 (Chomiuk et al. 2011; Quimby et al. 2011c), SLSNe reach optical-UV luminosities $L>7 \times 10^{43} \mathrm{erg} \mathrm{s}^{-1}, \sim 10-100$ more luminous than common $\mathrm{SNe}$, and are likely associated with the death of massive stars. The source of energy powering their exceptional energy release is still debated (e.g., Gal-Yam 2012). The proposed energy sources include: (i) radioactive decay of large amounts of freshly synthesized ${ }^{56} \mathrm{Ni}\left(M_{\mathrm{Ni}} \gtrsim 5 M_{\odot}\right)$, a signature of pairinstability explosions (as proposed for SN 2007bi, Gal-Yam et al. 2009); (ii) SN shock interaction with dense material in the environment (e.g., Smith \& McCray 2007; Chevalier \& Irwin 2011); and (iii) a magnetar central engine (e.g., Kasen \& Bildsten 2010; Woosley 2010; Nicholl et al. 2013). The narrow features $\left(v \lesssim 100 \mathrm{~km} \mathrm{~s}^{-1}\right)$ in the spectra of hydrogenrich SLSNe like SN 2006gy clearly indicate that the interaction

\footnotetext{
${ }^{10}$ Hubble, Carnegie-Dunlap Fellow.
}

of the SN blast wave with the medium plays a role (e.g., Ofek et al. 2007; Smith \& McCray 2007), while it is unclear if a single mechanism can power hydrogen-stripped SLSNe (i.e., SLSNe-I). Indeed, SLSN-I iPTF 13ehe has been interpreted as the combination of energy extracted from a magnetar central engine coupled with radiation from the radioactive decay of $\sim 2.5 M_{\odot}$ of ${ }^{56} \mathrm{Ni}$, and a late-time interaction of the $\mathrm{SN}$ shock with the medium (Yan et al. 2015; Wang et al. 2016b).

A number of independent lines of evidence support the idea that SLSNe-I might harbor an engine. Observations of SLSN-I host galaxies indicate a preference for low-metallicity environments, which inspired a connection with long gamma-ray bursts (GRBs, Lunnan et al. 2014; Leloudas et al. 2015b; Chen et al. 2017; Perley et al. 2016; see, however, Angus et al. 2016). Along the same line, Greiner et al. (2015) reported the detection of SN 2011kl associated with GRB 111209A (Kann et al. 2016) with color and luminosity properties that are reminiscent of SLSNe, and suggested that a magnetar central engine powered both the initial burst of $\gamma$-rays and the later optical/UV SN emission (Metzger et al. 2015). Milisavljevic et al. (2013) found links between the late-time emission properties of a subset of 
Table 1

Gold Sample

\begin{tabular}{lccccc}
\hline \hline SN & $z$ & $\begin{array}{c}d_{L} \\
(\mathrm{Mpc})\end{array}$ & $\begin{array}{c}\text { Discovery Date } \\
(\mathrm{MJD})\end{array}$ & $\begin{array}{c}\text { Inferred Explosion Date } \\
\text { (MJD) }\end{array}$ & $\begin{array}{c}\mathrm{NH}_{\mathrm{Mw}} \\
\left(10^{20} \mathrm{~cm}^{-2}\right)\end{array}$ \\
\hline SCP 06F6 & 1.189 & 8310 & $53787^{\mathrm{a}}$ & $53767^{\mathrm{b}}$ & 0.885 \\
PTF 12dam & 0.107 & 498 & $56037^{\mathrm{a}}$ & $56022^{\mathrm{c}}$ & $\begin{array}{c}\text { Instrument } \\
\text { PS1-14bj }\end{array}$ \\
SN 2015bn/PS15ae & 0.521 & 3012 & $56618^{\mathrm{d}}$ & $56611^{\mathrm{e}}$ & Swift+CXO \\
\hline
\end{tabular}

Notes.

${ }^{a}$ From Levan et al. (2013).

b The time of the peak is MJD 53872. The rise-time is $~ 50$ days in the rest-frame (Barbary et al. 2009).

c The light curve reached maximum light on MJD 56088 and the rest-frame rise-time is $\sim 60$ days (Nicholl et al. 2013).

${ }^{\mathrm{d}}$ From Lunnan et al. (2016).

e Lunnan et al. (2016) estimate a peak time on MJD 56801.3 and a rest-frame rise-time $\gtrsim 125$ days.

${ }^{\mathrm{f}}$ From Nicholl et al. (2016b).

$\mathrm{g}$ The SN reached $r$-band maximum light on MJD 57102 (Nicholl et al. 2016b). The rise-time inferred by Nicholl et al. (2016b) is $\sim 80$ days in the rest-frame.

energetic, slow-evolving supernovae, and the superluminous SN 2007bi. They suggested that a single, possibly jetted, explosion mechanism may unify all of these events that span $-21 \leqslant M_{B} \leqslant-17$ mag. ${ }^{11}$ Additionally, nebular spectroscopic studies by Nicholl et al. (2016b) revealed similarities between the SLSN-I 2015bn and SN 1998bw, associated with GRB 980425, suggesting that the cores of their massive progenitors shared a similar structure at the time of collapse. In another source, SLSNI Gaia 16apd, Nicholl et al. (2017) further demonstrated that the luminous excess of UV emission originates from a central source of energy, rather than reduced UV absorption or shock interaction with a thick medium (see, however, Yan et al. 2017). Finally, luminous X-ray emission has been detected at the location of the SLSN-I SCP 06F6 (Gänsicke et al. 2009) with a luminosity $L_{x} \sim 10^{45} \mathrm{erg} \mathrm{s}^{-1} \sim 70$ days (rest-frame) after the explosion (Levan et al. 2013). At this epoch, SCP 06F6 even outshines GRBs by a large factor, suggesting the presence of a still-active central engine that manifests itself through very luminous and long-lasting X-ray emission (Levan et al. 2013; Metzger et al. 2015). Before our efforts, SCP 06F6 was the only SLSN-I for which an X-ray source was detected at a location consistent with the optical transient.

These observational results suggest a connection between SLSNe-I and engine-driven SNe. However, it is not yet known how the properties of the engines (successful jet? relativistic ejecta? collimated or spherical central-engine-powered outflow?), progenitor stars, and circumstellar environments would compare. Here, we present the results from a systematic search for X-ray emission from SLSNe-I both at early and at late times, which directly depends on the properties of the immediate environment and central engine (if any). The direct detection of the stellar progenitors of SLSNe-I in pre-explosion optical images is not possible due to their large distances $(z \geqslant 0.1)$. Sampling the circumstellar density profile in the closest environment is thus the most direct probe of their progenitors and their recent mass-loss history before stellar death.

The data set that we present here includes the deepest X-ray observations of SLSNe-I with Swift, XMM, and the Chandra $X$-ray Observatory $(C X O)$, extending from the time of discovery until $\sim 2000$ days (rest-frame) after explosion, and

\footnotetext{
$11 \mathrm{~N}$ ote that Soker (2017 and references therein) go a step further and interestingly propose that all core-collapse $\mathrm{SNe}$ are in fact jet-driven explosions.
}

led to the discovery of X-ray emission at the location of the slowly evolving SLSN-I PTF 12dam. These observations, described in Section 2, indicate that superluminous X-ray emission similar to what was observed in association with SCP 06F6 is not common in SLSNe-I (Section 3) and allow us to place meaningful constraints on the environment density at the SLSN site (Section 4). We constrain the properties of central engines in SLSNe-I in Section 5 by investigating the presence of late-time X-ray re-brightenings that can either be due to emission from off-axis collimated relativistic outflows similar to GRBs, or to the ionization breakouts from magnetar central engines (Metzger et al. 2014). Conclusions are drawn in Section 6 .

\section{X-Ray Observations and Analysis}

Since 2011, we routinely followed up all publicly announced nearby $(z \lesssim 0.5)$ SLSNe-I with Swift-XRT with a series of observations acquired between the time of discovery and $~ 360$ days (rest-frame) after explosion. For a subset of events we acquired deep X-ray observations with dedicated programs on the $C X O$ and $X M M$-Newton. Additionally, we searched the Swift-XRT, $C X O$, and XMM archives for serendipitous or unpublished observations of SLSNe-I discovered before 2016 May. Our final sample consists of 26 SLSNe-I discovered between 2006 and 2016 May. The data set covers the time range between $\sim$ days after explosion until $\sim 2000$ days (restframe) and comprises $\sim 700 \mathrm{hr}$ of observations. We update the X-ray observations of the sample of 11 SLSNe-I from Levan et al. (2013) with the most recent data ${ }^{12}$ and we add 15 new SLSNe-I. Inserra et al. (2017) presented a selection of SwiftXRT observations of three SLSNe. The much longer temporal baseline and better sensitivity of the X-ray data set presented here allow us to constrain the environments and the properties of central engines possibly powering the SLSN emission.

We divide our sample into three groups: the "gold sample" (Table 1) contains 4 SLSNe-I with X-ray detections or wellsampled optical bolometric light curve and deep X-ray limits obtained with $X M M$ or the $C X O$. The "bronze sample" contains 12 SLSNe-I with sparser optical data but with good Swift-XRT

\footnotetext{
${ }^{12}$ Note that PTF 11dsf and CSS121015, included by Levan et al. (2013) in the sample of SLSNe-I, are in fact H-rich events (see Benetti et al. 2014; Perley et al. 2016). Additionally, for PTF 11 dsf an AGN interpretation cannot be ruled out (Perley et al. 2016). For these reasons, we do not include these two events in our sample of SLSNe-I.
} 
Table 2

Bronze Sample

\begin{tabular}{|c|c|c|c|c|c|c|}
\hline $\mathrm{SN}$ & $z$ & $\begin{array}{c}d_{L} \\
(\mathrm{Mpc})\end{array}$ & $\begin{array}{l}\text { Discovery Date } \\
\text { (MJD) }\end{array}$ & $\begin{array}{l}\text { Inferred Explosion Date } \\
\text { (MJD) }\end{array}$ & $\underset{\left(10^{20} \mathrm{~cm}^{-2}\right)}{\mathrm{NH}_{\mathrm{MW}}}$ & Instrument \\
\hline PTF 09cnd & 0.258 & 1317 & $55025^{\mathrm{a}}$ & $55006^{\mathrm{b}}$ & 2.20 & $\overline{\text { Swift }+X M M}$ \\
\hline SN 2010gx & 0.230 & 1156 & $55260^{\mathrm{a}}$ & $55251^{\mathrm{c}}$ & 3.28 & Swift \\
\hline SN $2011 \mathrm{ke}$ & 0.143 & 682 & $55650^{\mathrm{a}}$ & $55649^{\mathrm{e}}$ & 1.27 & Swift $+C X O$ \\
\hline SN 2012il & 0.175 & 851 & $55926^{\mathrm{a}}$ & $55919^{\mathrm{e}}$ & 2.38 & Swift \\
\hline iPTF 13ehe & 0.3434 & 1833 & $56621^{\mathrm{f}}$ & $56496.4^{\mathrm{g}}$ & 4.30 & Swift \\
\hline CSS140925-005854 & 0.46 & 2590 & $56920^{\mathrm{k}}$ & $56900^{\mathrm{k}}$ & 3.99 & Swift \\
\hline LSQ 14fxj & 0.36 & 1937 & $56942^{1}$ & $56872^{\mathrm{m}}$ & 3.28 & Swift \\
\hline DES15S2nr & 0.220 & 1099 & $57251^{\mathrm{n}}$ & $57251^{\circ}$ & 3.02 & Swift \\
\hline SN 2016ard/PS16aqv & $0.2025^{\mathrm{p}}$ & 988 & $57438^{\mathrm{q}}$ & $57393^{r}$ & 3.97 & Swift \\
\hline
\end{tabular}

Notes.

a From Levan et al. (2013).

${ }^{\mathrm{b}}$ From Quimby et al. (2011c), the peak time is MJD 55069.145 and the rest-frame rise-time is $\sim 50$ days.

${ }^{c}$ From Quimby et al. (2011c), the peak time is MJD 55279 and the rest-frame rise-time is $\sim 23$ days.

${ }^{\mathrm{d}}$ Vinko et al. (2012) report that SN 2010kd reached maximum light 40 days after discovery. We assume a 50 day rest-frame rise-time.

e From Inserra et al. (2013).

${ }^{\mathrm{f}}$ From Yan et al. (2015).

g Yan et al. (2015) report a range of explosion dates between MJD 56470.8 and MJD 56522.0. We use the middle date MJD 56496.4.

${ }^{\text {h }}$ From Leloudas et al. (2015a).

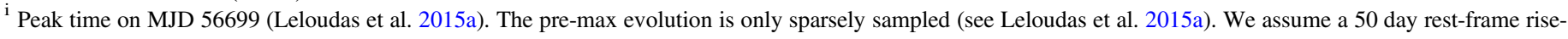
time, similar to other SLSNe-I.

j From Jerkstrand et al. (2017).

${ }^{\mathrm{k}}$ From the CRTS source catalog http://nesssi.cacr.caltech.edu/catalina/AllSN.arch.html

${ }^{1}$ From Smith et al. (2014).

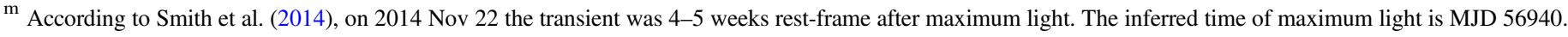
We assume a 50 days rest-frame rise-time.

n From D'Andrea et al. (2015).

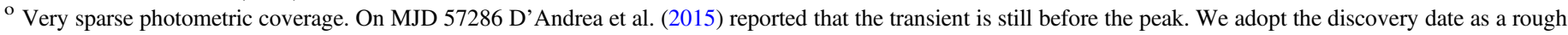
proxy for the explosion date here.

${ }^{\mathrm{p}}$ From P. Blanchard et al. (2018, in preparation).

${ }^{\mathrm{q}}$ From http://star.pst.qub.ac.uk/ps1threepi/psdb/public/.

${ }^{\mathrm{r}}$ The peak time is MJD 57453 from http://star.pst.qub.ac.uk/ps1threepi/psdb/public/. We assume a 50 day rest-frame rise-time.

X-ray coverage (Table 2), while the "iron sample" comprises 10 SLSNe-I with very sparse optical and X-ray data (Table 3). Given the peculiar nature of ASASSN-15lh (Dai et al. 2015; Metzger et al. 2015; Bersten et al. 2016; Chatzopoulos et al. 2016; Dong et al. 2016; Godoy-Rivera et al. 2017; Kozyreva et al. 2016; Leloudas et al. 2016; Sukhbold \& Woosley 2016; van Putten \& Della Valle 2017; Margutti et al. 2017a), this transient is not part of the sample of bona fide SLSNe-I analyzed here. However, we discuss and compare the X-ray properties of ASASSN-15lh in the context of SLSNe-I in Section 4, 5.1 and 5.2.

Swift-XRT data have been analyzed using HEASOFT (v6.18) and corresponding calibration files, following standard procedures (see Margutti et al. 2013b for details). For each SLSN-I we provide stacked flux limits (for visualization purposes only), and flux limits derived from individual observations (see Table 5 in the Appendix). CXO data have been analyzed with the CIAO software package (v4.9) and corresponding calibration files. Standard ACIS data filtering has been applied. $X M M$ data have been analyzed with SAS (v15.0). For the non-detections, we perform a flux calibration adopting a power-law spectral model with index $\Gamma=2$ corrected for the Galactic neutral-hydrogen absorption along the line of sight (Tables 1-3), as inferred from Kalberla et al.
(2005). The details of the X-ray observations of specific SLSNe-I are provided in Section 2.1 for the gold sample, and in the Appendix for all the other SLSNe-I. Data tables can also be found in the Appendix. Figure 1 shows the complete sample of X-ray observations of SLSNe-I.

\subsection{Gold Sample}

There are four objects in the gold sample: SCP 06F6, PTF 12dam, PS1-14bj, and SN 2015bn (Table 1). In this section we describe the discovery and properties of each object in the gold sample.

\subsubsection{SCP $06 F 6$}

$\mathrm{X}$-ray emission at the location of the type-I SLSN SCP 06F6 was first reported by Gänsicke et al. (2009) from XMM observations obtained 162 days after the initial detection of SCP 06F6 (PI Shartel, ID 0410580301). Levan et al. (2013) derived an X-ray flux $F_{x} \sim 10^{-13} \mathrm{erg} \mathrm{s}^{-1} \mathrm{~cm}^{-2}(0.3-10 \mathrm{keV})$ on 2006 August 2 (MJD 53949, 80 days rest-frame since explosion). Given the importance of the claim, we independently reanalyzed the $X M M$ observations of SCP $06 \mathrm{~F} 6$. We confirm the presence of severe contamination by soft proton flares and we confirm the detection of a point-like X-ray source 
Table 3

Iron Sample

\begin{tabular}{|c|c|c|c|c|c|c|}
\hline $\mathrm{SN}$ & $z$ & $\begin{array}{c}d_{L} \\
(\mathrm{Mpc})\end{array}$ & $\begin{array}{l}\text { Discovery Date } \\
\text { (MJD) }\end{array}$ & $\begin{array}{l}\text { Inferred Explosion Date } \\
\text { (MJD) }\end{array}$ & $\underset{\left(10^{20} \mathrm{~cm}^{-2}\right)}{\mathrm{NH}_{\mathrm{MW}}}$ & Instrument \\
\hline SN 2009jh/PTF 09cwl & 0.349 & 1868 & $55010^{\mathrm{a}}$ & $55010^{\mathrm{b}}$ & 1.49 & Swift \\
\hline PTF 09atu & 0.501 & 2870 & $55016^{\mathrm{a}}$ & $54988^{c}$ & 3.79 & Swift \\
\hline PTF 10aagc & 0.207 & 1027 & Unclear & $55413^{\mathrm{d}}$ & 2.61 & Swift \\
\hline SN 2010md/PTF 10hgi & 0.098 & 463 & $55331^{\mathrm{a}}$ & $55323^{\mathrm{e}}$ & 5.81 & Swift \\
\hline PS1-11bdn & 0.738 & 4601 & $55910.4^{\mathrm{f}}$ & $55889.2^{\mathrm{f}}$ & 3.76 & Swift \\
\hline PTF 11rks & 0.19 & 933 & $55916^{\mathrm{a}}$ & $55912^{\mathrm{e}}$ & 4.66 & Swift \\
\hline DES15C3hav & 0.392 & 2142 & $57310^{\mathrm{g}}$ & $57270^{\mathrm{h}}$ & 0.705 & Swift \\
\hline OGLE15qz & 0.63 & 3790 & 57264 & $57264^{\mathrm{i}}$ & 4.28 & Swift \\
\hline OGLE15sd & 0.656 & 3319 & 57295 & $57295^{\mathrm{i}}$ & 9.44 & Swift \\
\hline PS16op & 0.48 & 2726 & $57398^{\mathrm{j}}$ & $57323^{\mathrm{k}}$ & 6.73 & Swift \\
\hline
\end{tabular}

Notes.

${ }^{a}$ From Levan et al. (2013).

b The time of the peak is MJD 55081 and the rise-time is $\sim 50$ days in the rest-frame (Quimby et al. 2011c).

c The time of maximum light is MJD 55063 (Quimby et al. 2011c). We assume a 50 day rise-time in the rest-frame.

${ }^{\mathrm{d}}$ The time of maximum light is MJD 55473 (Perley et al. 2016). We assume a 50 day rise-time in the rest-frame.

e From Inserra et al. (2013).

${ }^{\mathrm{f}}$ From R. Lunnan et al. (2018, in preparation).

g From Challis et al. (2016).

${ }^{\mathrm{h}}$ From Challis et al. (2016) the peak time is MJD 57340. We assume a 50 day rest-frame rise-time.

${ }^{\mathrm{i}}$ From http://ogle.astrouw.edu.pl/ogle4/transients/transients.html.

${ }^{\mathrm{j}}$ From Dimitriadis et al. (2016).

${ }^{\mathrm{k}}$ The peak time is MJD 57397 (Dimitriadis et al. 2016). We assume a 50 day rest-frame rise-time.

in the $0.2-2 \mathrm{keV}$ energy range in MOS1 and MOS2. The source is detected at the $\sim 5 \sigma$ confidence level at coordinates R.A. $=14^{\mathrm{h}} 32^{\mathrm{m}} 27^{\mathrm{s}} .586$, decl. $=33^{\circ} 32^{\prime} 25^{\prime \prime} 33(\mathrm{~J} 2000)$, consistent within the $4^{\prime \prime}$ position error with the optical position of SCP 06F6. The 0.3-10 keV flux inferred from the count rate $(12.8 \pm 2.3) \times 10^{-3} \mathrm{cts} \mathrm{s}^{-1}$ is in agreement with the findings from Levan et al. (2013).

Follow-up observations of SCP 06F6 with the $C X O$ obtained on 2006 November 4 (MJD 54043, 126 days rest-frame since explosion, PI Murray, ID 7010) led to a non-detection. The corresponding flux limit is $F_{x}<1.4 \times 10^{-14} \mathrm{erg} \mathrm{s}^{-1} \mathrm{~cm}^{-2}$ (Levan et al. 2013). We adopt these flux values here and the correct redshift for this event, which is $z=1.189$ (Quimby et al. 2011c).

\subsubsection{X-Ray Emission at the Location of PTF 12dam}

PTF 12dam (Nicholl et al. 2013; Chen et al. 2015; Inserra et al. 2017; Vreeswijk et al. 2017) belongs to the small subset of SLSNe-I with slowly evolving optical light curves. At the time of writing, this group includes SN 2007bi, PTF 12dam, iPTF 13ehe, SN 2015bn, PS1-14bj, and LSQ 14an. The slow evolution of these transients, and of SN 2007bi in particular, inspired a connection with pair-instability explosions (Gal-Yam et al. 2009) that was later debated by Nicholl et al. (2013). X-ray observations of the SLSN-I PTF 12dam have been obtained with Swift-XRT and the CXO. Swift-XRT observations span the time range of $\sim 43-900$ days rest-frame since explosion and revealed no detection down to a flux limit $F_{x} \sim 5 \times 10^{-14} \mathrm{erg} \mathrm{s}^{-1} \mathrm{~cm}^{-2}$ (Figure 2, Table 5).

A set of three deep CXO observations was acquired between 2012 June 11 and 19 ( $\delta t \sim 60-68$ days rest-frame since explosion; observations IDs 13772, 14444 and 14446, PI Pooley). An X-ray source with a soft spectrum is clearly detected in the merged event file (total exposure of $99.9 \mathrm{ks}$ ) at the location of PTF $12 \mathrm{dam}$ with significance of $4.8 \sigma$ in the
$0.5-2 \mathrm{keV}$ energy range. The measured net count rate is $(7.1 \pm 2.8) \times 10^{-5} \mathrm{c} \mathrm{s}^{-1}(0.5-2 \mathrm{keV})$, which corresponds to an unabsorbed flux of $(7.3 \pm 2.9) \times 10^{-16} \mathrm{erg} \mathrm{s}^{-1} \mathrm{~cm}^{-2}$ $(0.3-10 \mathrm{keV})$ assuming a power-law spectrum with photon index $\Gamma=2$. For a thermal bremsstrahlung spectrum with $T=0.24 \mathrm{keV}$ (see below) the corresponding unabsorbed flux is $(8.9 \pm 3.5) \times 10^{-16} \mathrm{erg} \mathrm{s}^{-1} \mathrm{~cm}^{-2}(0.3-10 \mathrm{keV})$, and $(3.5 \pm 1.4) \times 10^{-16} \mathrm{erg} \mathrm{s}^{-1} \mathrm{~cm}^{-2}(0.5-2 \mathrm{keV})$. In both cases, the inferred X-ray luminosity is $L_{x} \sim 2 \times 10^{40} \mathrm{erg} \mathrm{s}^{-1}$ in the $0.3-10 \mathrm{keV}$ (Figure 2).

PTF 12dam exploded in a compact dwarf galaxy with fairly large star formation rate SFR $\sim 5 M_{\odot} \mathrm{yr}^{-1}$ (Lunnan et al. 2014; Chen et al. 2015; Leloudas et al. 2015b; Thöne et al. 2015; Perley et al. 2016) Following Mineo et al. (2012), the expected apparent diffuse X-ray emission associated with star formation is $L_{x} / \mathrm{SFR} \approx 8.3 \times 10^{38} \mathrm{erg} \mathrm{s}^{-1}\left(M_{\odot} \mathrm{yr}^{-1}\right)^{-1}$, which translates into $L_{x} \approx 4.2 \times 10^{39} \mathrm{erg} \mathrm{s}^{-1}(0.5-2 \mathrm{keV})$ for SFR $\sim 5 M_{\odot} \mathrm{yr}^{-1}$. As a comparison, for a thermal bremsstrahlung spectrum with $T=0.24 \mathrm{keV}$ (average temperature of the unresolved X-ray component in galaxies, Mineo et al. 2012), for PTF 12dam we calculate $L_{x} \approx(1.0 \pm 0.4) \times 10^{40} \mathrm{erg} \mathrm{s}^{-1} \quad(0.5-2 \mathrm{keV})$. We therefore conclude that star formation in the host galaxy of PTF 12dam is likely contributing to at least some of the X-ray luminosity that we detected at the location of the transient. In the following analysis sections we treat our measurements as upper limits to the X-ray emission from PTF 12dam. These observations provide the deepest limits to the X-ray emission from a SLSN-I to date. Future observations will constrain the late-time behavior of the X-ray emission at the location of PTF 12dam and will clarify its association to the optical transient.

\subsubsection{PS1-14bj}

Two epochs of deep X-ray observations of the type-I SLSN PS1-14bj (Lunnan et al. 2016) have been obtained with XMM 


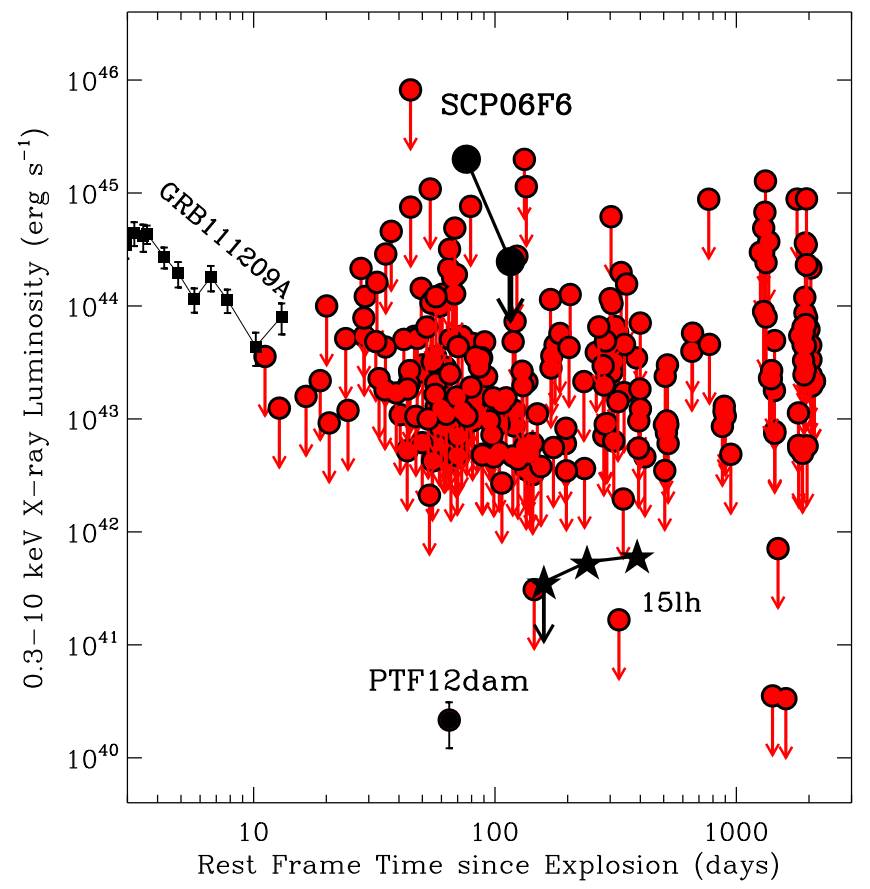

Figure 1. X-ray observations of SLSNe-I spanning the time range $\sim 10-2000$ days (red circles for upper limits, black circles for detections) show that superluminous X-ray emission of the kind detected at the location of SCP 06F6 (Gänsicke et al. 2009; Levan et al. 2013) is not common. Black stars: X-ray emission at the location of ASASSN-15lh (Margutti et al. 2017b), which has a very disputed physical origin. Black squares: X-ray afterglow of GRB 112109A, associated with the overluminous SN 2011kl (Greiner et al. 2015).

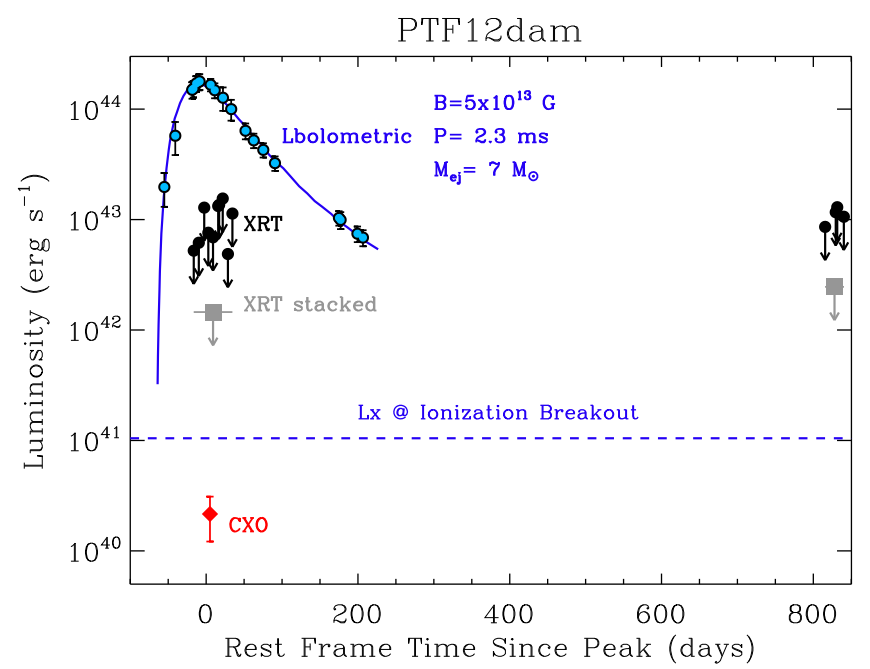

Figure 2. Deep $C X O$ observations (red diamond) obtained around the time of the optical peak reveal the presence of soft X-ray emission at the location of PTF 12dam, with luminosity $L_{x} \sim 2 \times 10^{40} \mathrm{erg} \mathrm{s}^{-1}$. Black filled circles: X-ray luminosity limits from Swift-XRT. Gray filled squares: stacked limits from Swift-XRT observations. Blue filled circles: bolometric optical emission as computed by Nicholl et al. (2013). Blue solid line: best-fitting magnetar model from Nicholl et al. (2013) with parameters reported in Table 4. Horizontal blue dashed line: X-ray luminosity at the time of ionization breakout according to the equations in Section 5.2 for the best-fitting magnetar parameters (Table 4). The expected time of ionization breakout is $t_{\text {ion }}=4.7 \mathrm{yr}$; see Table 4 .

(PI Margutti, IDs 0743110301, 0743110701) on 2014 June 9 $(\delta t \sim 135$ days rest-frame since explosion, exposure time of $47.6 \mathrm{ks}$ ), and 2014 November 7 ( $\delta t \sim 235$ days rest-frame

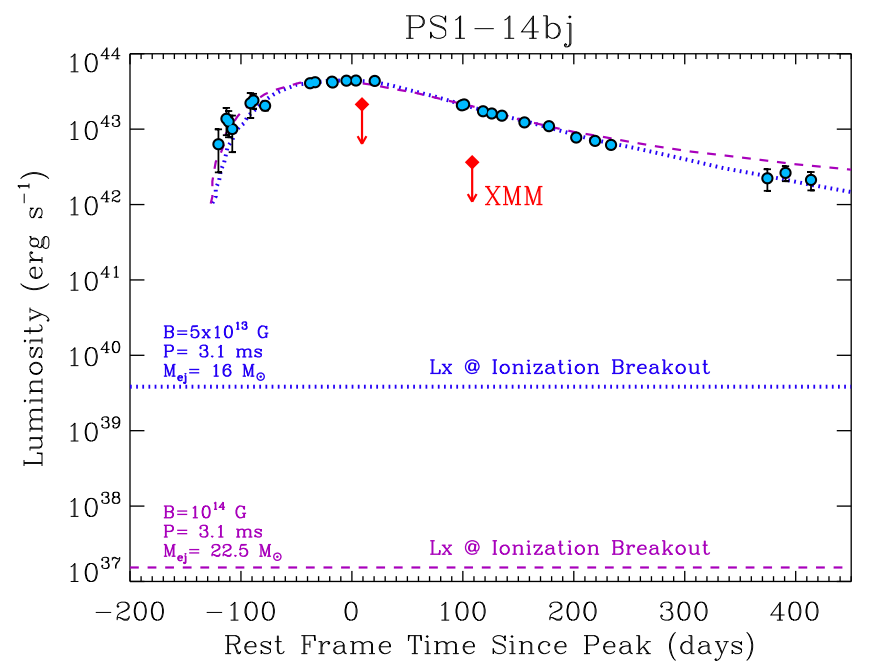

Figure 3. Luminosity limits on the X-ray emission from PS1-14bj obtained with $X M M$ (red diamonds). Blue filled circles: bolometric luminosity as computed by Lunnan et al. (2016). Blue dotted line and purple dashed line: magnetar models that adequately fit the observations as computed by Lunnan et al. (2016; see Table 4). Horizontal lines: X-ray luminosity at the time of ionization breakout according to the equations in Section 5.2 for the two magnetar models. For these models, the expected time of ionization breakout is $t_{\text {ion }} \geqslant 25$ years; see Table 4 .

since explosion, exposure of $36.0 \mathrm{ks}$ ). The net exposure times after removing data with high background contamination are $3.6 \mathrm{ks}$ and $29.8 \mathrm{ks}$, respectively (EPIC-pn data). We do not find evidence for significant X-ray emission at the location of PS1$14 \mathrm{bj}$ in either observation and derive a $3 \sigma 0.3-10 \mathrm{keV}$ countrate upper limit of $9.4 \times 10^{-3} \mathrm{c} \mathrm{s}^{-1}\left(1.5 \times 10^{-3} \mathrm{c} \mathrm{s}^{-1}\right)$ for the first (second) epoch, which translates into an unabsorbed flux of $1.9 \times 10^{-14} \mathrm{erg} \mathrm{s}^{-1} \mathrm{~cm}^{-2}\left(3.3 \times 10^{-15} \mathrm{erg} \mathrm{s}^{-1} \mathrm{~cm}^{-2}\right)$. The corresponding luminosity limits are shown in Figure 3 and reported in Table 5. Analogous with the type of analysis performed on SCP 06F6, we also inspected the $0.2-2 \mathrm{keV}$ images (for both EPIC-pn and MOS) without filtering for the flares. However, for PS1-14bj we confirm the non-detection in both epochs.

\subsubsection{SN $2015 b n$}

X-ray observations of the SLSN-I 2015bn (Nicholl et al. 2016a, 2016b) have been obtained with Swift-XRT and XMM (PI Margutti, IDs 0770380201, 0770380401). A first set of observations was presented in Nicholl et al. (2016a), while Inserra et al. (2017) included in their analysis five Swift-XRT pointings. Here, we present the complete data set. Swift-XRT started observing SN 2015bn on 2015 February 19 until 2016 July 23, covering the time period $\sim 44-522$ days since explosion rest-frame. No statistically significant X-ray emission is blindly detected at the location of the transient (Figure 4). ${ }^{13}$

\footnotetext{
${ }^{13}$ We note the presence of marginally significant ( $2 \sigma$ c.l.) soft X-ray emission (i.e., $<0.3 \mathrm{keV}$ ) with $L_{x} \sim 5 \times 10^{42} \mathrm{erg} \mathrm{s}^{-1}$ found in a targeted search of data acquired on 2015 February 22 (i.e., 55 days since explosion, rest-frame). However, emission with this flux is ruled out by Swift-XRT observations obtained $24 \mathrm{hr}$ before, and is not detected in Swift-XRT data with similar exposure time collected in the days afterward. Furthermore, we find no evidence for X-ray emission when we filter the event file in the standard $0.3-10 \mathrm{keV}$ energy range, which is where the Swift-XRT is properly calibrated. We conclude that the association of the targeted detection with real X-ray emission from SLSN-I 2015bn/PS15ae is highly questionable and therefore proceed with the conclusion that there is no statistically significant X-ray emission at the location of the transient.
} 


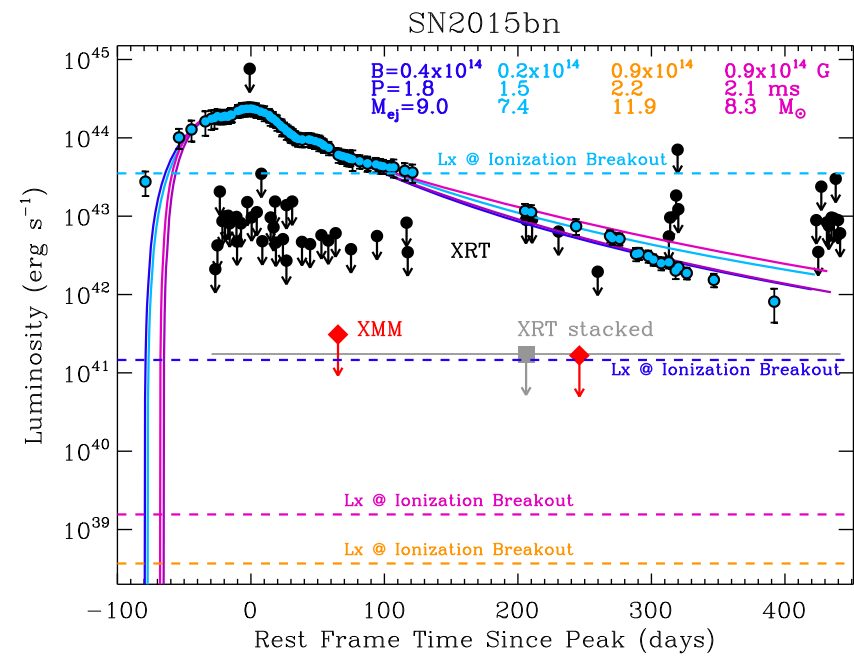

Figure 4. Results from our joint Swift-XRT and XMM X-ray campaign (black filled circles, gray squares and red diamonds) in the context of the optical bolometric luminosity of SN 2015bn and the best-fitting magnetar models as derived by Nicholl et al. (2016b; see Table 4). Horizontal dashed lines: expected X-ray luminosity at the time of the ionization breakout, which is $t_{\text {ion }}=5 \mathrm{yr}\left(\right.$ for $\left.B=0.4 \times 10^{14} \mathrm{G}, P=1.8 \mathrm{~ms}, M_{\mathrm{ej}}=9 M_{\odot}\right), t_{\text {ion }}=0.6 \mathrm{yr}$ (for $B=0.2 \times 10^{14} \mathrm{G}, \quad P=1.5 \mathrm{~ms}, \quad M_{\mathrm{ej}}=7.4 M_{\odot}$ ), $t_{\mathrm{ion}}=45 \mathrm{yr} \quad$ (for $\left.B=0.9 \times 10^{14} \mathrm{G}, \quad P=2.2 \quad \mathrm{~ms}, \quad M_{\mathrm{ej}}=11.9 M_{\odot}\right), \quad t_{\text {ion }}=22 \mathrm{yr} \quad$ (for $\left.B=0.9 \times 10^{14} \mathrm{G}, P=2.1 \mathrm{~ms}, M_{\mathrm{ej}}=8.3 M_{\odot}\right)$ as reported in Table 4 . The models with the shortest spin periods are disfavored by our X-ray limits. This figure clearly shows how magnetar models associated with very similar bolometric optical light curves do predict instead very different X-ray luminosities at ionization breakout. The X-ray luminosity at the time of breakout is a very sensitive probe of the properties of central engines in SLSNe.

Two epochs of $X M M$ observations have been obtained on 2015 June 1 ( $\delta t \sim 145$ days rest-frame since explosion) and 2015 December 18 ( $\delta t \sim 325$ days rest-frame since explosion) with exposure times of $28.0 \mathrm{ks}$ and $25.1 \mathrm{ks}$, respectively (EPICpn data). After excluding time intervals heavily affected by proton flaring, the net exposure times are 7.3 and $18.8 \mathrm{ks}$. No X-ray source is detected at the location of the SLSN-I $2015 \mathrm{bn}$. We derive a $3 \sigma \quad 0.3-10 \mathrm{keV}$ count-rate upper limit of $4.2 \times 10^{-3} \mathrm{c} \mathrm{s}^{-1}\left(2.3 \times 10^{-3} \mathrm{c} \mathrm{s}^{-1}\right)$ for the first (second) epoch, which translates into an unabsorbed flux of $9.8 \times 10^{-15} \mathrm{erg} \mathrm{s}^{-1} \mathrm{~cm}^{-2} \quad\left(5.3 \times 10^{-15} \mathrm{erg} \mathrm{s}^{-1} \mathrm{~cm}^{-2}\right) . \quad$ The results from our $\mathrm{X}$-ray campaign are listed in Table 5 and displayed in Figure 4.

\section{Search for Superluminous X-Ray Emission in SLSNe-I}

In this section we derive constraints on the possible presence of superluminous X-ray emission in SLSNe-I that was not detected because of the discontinuous observational coverage. No assumption is made about the physical nature of the emission. SLSNe-I are treated here as different realizations of the same stochastic process (which is the underlying assumption behind any sample analysis).

The hypothesis we test is that superluminous X-ray emission of the kind detected at the location of SCP 06F6 (i.e., isotropic $L_{x} \sim 10^{45} \mathrm{erg} \mathrm{s}^{-1}$ ) is ubiquitous in SLSNe-I. We furthermore assume that the superluminous X-ray emission is "on" for a total time $\Delta t_{\text {active }}$ (not necessarily continuous) and shuts on and off instantaneously, and that the probability of turning on is uniformly distributed during the interval of time of investigation. Our sample of observations comprises 253 spacecraft pointings, for a total observing time of $\sim 30$ days at $t<2000$ days (rest-frame). Out of 253 trials, observations only led to one success (i.e., in the case of SCP 06F6). We can use simple binomial probability arguments to constrain the maximum and minimum $\Delta t_{\text {active }}$ that would be statistically consistent at the $3 \sigma$ c.l. with $\leqslant 1$ successes (for $\Delta t_{\text {active, } \max }$ ) and $\geqslant 1$ successes (for $\left.\Delta t_{\text {active,min }}\right)$ out of $N$ trials, where $N \equiv N(t)$. In this context a trial consists of an observation that is deep enough to be sensitive to $L_{x} \sim 10^{45} \mathrm{erg} \mathrm{s}^{-1}$. For each trial, the probability of success is $p=\Delta t_{\text {active }} / \Delta t_{\text {total }}$, where $\Delta t_{\text {total }}$ is the entire range of times during which we conduct our search for superluminous X-ray emission (for the entire sample, $\Delta t_{\text {total }} \sim 2000$ days). For $t<100$ days, $\Delta t_{\text {total }} \sim 90$ days (i.e., our search starts at $t \sim 10$ days), $N=110$. With these parameters we find $200 \mathrm{~s} \leqslant \Delta t_{\text {active }} \leqslant 5 \times 10^{5} \mathrm{~s}$. For shorter $\Delta t_{\text {active }}$ the probability of having one success out of $N$ trials is below the $3 \sigma$ c.l., while for longer $\Delta t_{\text {active }}$ we would have expected to have more successes in our search at $3 \sigma$ c.l. If we consider the entire sample of observations $\Delta t_{\text {total }} \sim 2000$ days, $N=253$ and we find $2000 \mathrm{~s} \leqslant \Delta t_{\text {active }} \leqslant 5 \times 10^{6} \mathrm{~s}$. As a cross-check, under the same assumptions, but adopting the Bayesian technique of Romano et al. (2014) we obtain for $\Delta t_{\text {active }}$ similar upper limits.

We conclude that superluminous X-ray emission is not a common trait of SLSNe-I. If present, the superluminous X-ray emission requires peculiar physical circumstances to manifest and its duration is $\leqslant 2$ months at $t<2000$ days and $\leqslant$ few days at $t<100$ days.

\section{Constraints on SLSNe-I Environments}

Inverse Compton (IC) emission is a well-known source of $\mathrm{X}$-rays in young stellar explosions (Björnsson \& Fransson 2004; Chevalier \& Fransson 2006). X-ray emission originates from the up-scattering of optical photons from the $\mathrm{SN}$ photosphere by a population of relativistic electrons accelerated at the shock front. While always present, IC is the dominant emission mechanism at early times $(t \leqslant$ optical peak) for $\mathrm{SNe}$ propagating into low-density media. In the case of strong SN shock interaction with the medium, the dominant X-ray emission mechanism is instead bremsstrahlung (Björnsson \& Fransson 2004; Chevalier \& Fransson 2006), as it is indeed observed in type-IIn SNe and as recently confirmed by the first broadband X-ray spectra of strongly interacting SNe (Ofek et al. 2014; Margutti et al. 2017a). The analysis of the optical emission from SLSNe-I in the context of the interaction model (e.g., Nicholl et al. 2014, 2016 b) suggests that if SLSNe-I are powered by interaction, then the shock breaks out around the time of optical maximum light and the medium consists of a thick shell confined to small radii ( $R \sim 5 \times 10^{15} \mathrm{~cm}$ for SN 2015bn) surrounded by a lower density region. This conclusion is consistent with the lack of observed narrow lines in the optical spectra of SLSNe-I (in sharp contrast to ordinary and superluminous type-IIn $\mathrm{SNe}$ ): the presence of an extended unshocked region of dense circumstellar medium (CSM) would likely imprint low-velocity features that are not observed in SLSNe-I (see also Chevalier \& Irwin 2011). The X-ray observations that we will use in this section have been obtained at the time of maximum light or later, which is after the shock has broken out from the thick shell of material if a shell is there. In the following, we thus constrain the density around SLSNe-I under the conservative assumption that IC is the only source of X-ray radiation. Since we sample the time range $t>t_{\text {peak }}$, our density limits apply to the region $R \gtrsim 10^{16} \mathrm{~cm}$. 
Table 4

Magnetar Parameters (Magnetic Field $B$, Spin Period $P_{i}$, and Ejecta Mass $M_{\mathrm{ej}}$ ), Estimated from the Bolometric Optical Emission, and Corresponding Ionization Breakout Times $t_{\text {ion }}$ and X-Ray Luminosities $L_{x}\left(t_{\text {ion }}\right)$

\begin{tabular}{|c|c|c|c|c|c|c|}
\hline $\mathrm{SN}$ & $\begin{array}{c}B \\
(\mathrm{G})\end{array}$ & $\begin{array}{c}P_{i} \\
(\mathrm{~ms})\end{array}$ & $\begin{array}{c}M_{\mathrm{ej}} \\
\left(M_{\odot}\right)\end{array}$ & References & $\begin{array}{c}t_{\text {ion }} \\
\text { (years) }\end{array}$ & $\begin{array}{c}L_{x}\left(t_{\text {ion }}\right) \\
\left(\mathrm{erg} \mathrm{s}^{-1}\right)\end{array}$ \\
\hline SN 2010md/PTF 10hgi & $3.6 \times 10^{14}$ & 7.2 & 3.9 & Inserra et al. (2013) & 76.3 & $7.8 \times 10^{36}$ \\
\hline SN 2010gx & $7.4 \times 10^{14}$ & 2.0 & 7.1 & Inserra et al. (2013) & 1070 & $9.4 \times 10^{33}$ \\
\hline SN 2011ke & $6.4 \times 10^{14}$ & 1.7 & 8.6 & Inserra et al. (2013) & 1170 & $1.0 \times 10^{34}$ \\
\hline PTF 12dam & $5 \times 10^{13}$ & 2.3 & 7 & Nicholl et al. (2013) & 4.7 & $1.0 \times 10^{41}$ \\
\hline SN 2012il & $4.1 \times 10^{14}$ & 6.1 & 2.3 & Inserra et al. (2013) & 34.5 & $3.0 \times 10^{37}$ \\
\hline PS1-14bj & $5 \times 10^{13}$ & 3.1 & 16 & Lunnan et al. (2016) w. leakage & 24.8 & $3.8 \times 10^{39}$ \\
\hline \multirow[t]{5}{*}{ SN 2015bn/PS15ae } & $0.9 \times 10^{14}$ & 2.1 & 8.4 & Nicholl et al. (2016b) & 22.1 & $1.5 \times 10^{39}$ \\
\hline & $10^{14}$ & 1.7 & 15.1 & Nicholl et al. (2016b) & 88.4 & $7.6 \times 10^{37}$ \\
\hline & $0.9 \times 10^{14}$ & 2.1 & 8.3 & Nicholl et al. (2016a) & 21.6 & $1.6 \times 10^{39}$ \\
\hline & $0.2 \times 10^{14}$ & 1.5 & 7.4 & Nicholl et al. (2016a) & 0.64 & $3.5 \times 10^{43}$ \\
\hline & $0.9 \times 10^{14}$ & 2.2 & 11.9 & Nicholl et al. (2016a) & 44.5 & $3.7 \times 10^{38}$ \\
\hline
\end{tabular}

The X-ray emission from IC depends on (i) the density structure of the SN ejecta and of the CSM, (ii) the details of the electron distribution responsible for the up-scattering, (iii) the explosion parameters (ejecta mass $M_{\mathrm{ej}}$ and kinetic energy $E_{k}$ ), and (iv) the availability of seed optical photons $\left(L_{x, \text { IC }} \propto L_{\mathrm{bol}}\right.$, where $L_{\mathrm{bol}}$ is the bolometric optical luminosity). We employ the formalism of Margutti et al. (2012) modified to reflect the stellar structure of massive stars as in Margutti et al. (2014). We further assume a wind-like medium with $\rho_{\mathrm{CSM}} \propto R^{-2}$ as appropriate for massive stars, a power-law electron distribution $n_{e}(\gamma)=n_{0} \gamma^{-p}$ with $p \sim 3$ as indicated by radio observations of $\mathrm{H}$-stripped corecollapse SNe (Chevalier \& Fransson 2006) and a fraction of post-shock energy into relativistic electrons $\epsilon_{e}=0.1$ (e.g., Chevalier \& Fransson 2006). Since $L_{x, \mathrm{IC}} \propto L_{\mathrm{bol}}$, it is clear that the tightest constraints on $\rho_{\mathrm{CSM}}$ will be derived from the most nearby SLSNe-I, which have very bright optical emission and deep X-ray limits (i.e., they have the largest flux ratio $F_{\mathrm{opt}} / F_{x}$ constrained by observations). To this end, we analyze below the SLSNe-I 2015bn and PTF 12dam. We also provide constraints for the peculiar transient ASASSN-15lh.

For SN 2015bn we follow Nicholl et al. (2016a) and Nicholl et al. (2016b) and adopt a range of ejecta masses $M_{\mathrm{ej}}=7-15 M_{\odot}$ (Table 4). With these parameters and the optical bolometric light curve from Nicholl et al. (2016b; Figure 4), our X-ray nondetections constrain the pre-explosion mass-loss rate from the stellar progenitor of SN2015bn to $\dot{M}<10^{-2} M_{\odot} \mathrm{yr}^{-1}$ $\left(\dot{M}<10^{-1} M_{\odot} \mathrm{yr}^{-1}\right)$ for $E_{k}=10^{52} \mathrm{erg}\left(E_{k}=10^{51} \mathrm{erg}\right)$ and wind velocity $v_{w}=1000 \mathrm{~km} \mathrm{~s}^{-1}$, (Figures 5, 6), which is $\dot{M}<10^{-4} M_{\odot} \mathrm{yr}^{-1}\left(\dot{M}<10^{-3} M_{\odot} \mathrm{yr}^{-1}\right)$ for wind velocity $v_{w}=10 \mathrm{~km} \mathrm{~s}^{-1}$. In this context, the analysis of the radio observations of SN 2015bn indicates $\dot{M}<10^{-2} M_{\odot} \mathrm{yr}^{-1}$ for $v_{w}=10 \mathrm{~km} \mathrm{~s}^{-1}$ at $R>10^{15} \mathrm{~cm}$, while $\dot{M} \sim 10^{-2} M_{\odot} \mathrm{yr}^{-1}$ would be needed to explain the late-time optical light curve of the transient through continued ejecta-CSM interaction (Nicholl et al. 2016b). The X-ray analysis thus argues against the presence of an extended CSM region if $E_{k}>10^{51} \mathrm{erg}$ (as is likely the case) and suggests that another source of energy is powering the light curve after the peak. This result is consistent with the conclusions by Nicholl et al. (2016a): based on the spectroscopic similarity of SN 2015bn with the GRB SN 1998bw in the nebular phase,
Nicholl et al. (2016a) concluded that a central engine is driving the explosion.

For PTF 12dam we use $M_{\mathrm{ej}}=7 M_{\odot}$, as inferred by Nicholl et al. (2013) from the modeling of the optical bolometric emission (Table 4). We detect an X-ray source at the location of PTF 12dam with $L_{x} \sim 2 \times 10^{40} \mathrm{erg} \mathrm{s}^{-1}$. We treat this value as an upper limit to the X-ray luminosity from the transient to account for possible contamination from the host galaxy. For these values of the explosion parameters and the measured $L_{x}$, the inferred mass-loss rate is $\dot{M}<2 \times 10^{-5} M_{\odot} \mathrm{yr}^{-1}$ $\left(\dot{M}<4 \times 10^{-6} M_{\odot} \mathrm{yr}^{-1}\right)$ for $E_{k}=10^{51} \mathrm{erg}\left(E_{k}=10^{52} \mathrm{erg}\right)$ and $v_{w}=1000 \mathrm{~km} \mathrm{~s}^{-1}$ (Figures 5, 6). These are the tightest constraints to the pre-explosion mass-loss history of SLSNe-I progenitors.

If the peculiar transient ASASSN-15lh is associated with an $E_{k}=10^{52} \mathrm{erg}$ explosion with ejecta mass $M_{\mathrm{ej}}=5-10 M_{\odot}$ (Metzger et al. 2015; Bersten et al. 2016; Chatzopoulos et al. 2016; Dai et al. 2016; Dong et al. 2016; Kozyreva et al. 2016; Sukhbold \& Woosley 2016), the X-ray observations from Margutti et al. (2017b) imply $\dot{M}<5 \times 10^{-6} M_{\odot} \mathrm{yr}^{-1}$ $\left(\dot{M}<5 \times 10^{-5} M_{\odot} \mathrm{yr}^{-1}\right)$ for $v_{w}=1000 \mathrm{~km} \mathrm{~s}^{-1}$ for a thermal (non-thermal) X-ray spectrum. These values are typical of mass-loss rates from $\mathrm{H}$-stripped compact massive stars (Figures 5 and 6).

From our analysis, we conclude that for PTF 12dam the inferred limits rule out the densest environments that characterize type-IIn SNe (Figure 5), indicating that the strong SN shock interaction with an extended medium is unlikely to be the primary source of energy sustaining the very luminous display. Our data, however, do not constrain the presence of a dense medium confined to $d<10^{16} \mathrm{~cm}$, which might be the result of massive shell ejections by the stellar progenitor in the $\sim 10 \mathrm{yr}$ before collapse (for a shell ejection velocity of $1000 \mathrm{~km} \mathrm{~s}^{-1}$ ). Interestingly, a low-density environment with $\dot{M} \sim 4 \times 10^{-6} M_{\odot} \mathrm{yr}^{-1}$ was also inferred from radio and X-ray observations of the type-Ib SN 2012au (Kamble et al. 2014), which showed spectroscopic similarities with SLSN-I (Milisavljevic et al. 2013). The tight constraints obtained for PTF 12dam point to a clean environment, and argue against the dense CSM typical of extended progenitors like RSG stars. This result suggests that at least some SLSN-I progenitors are 


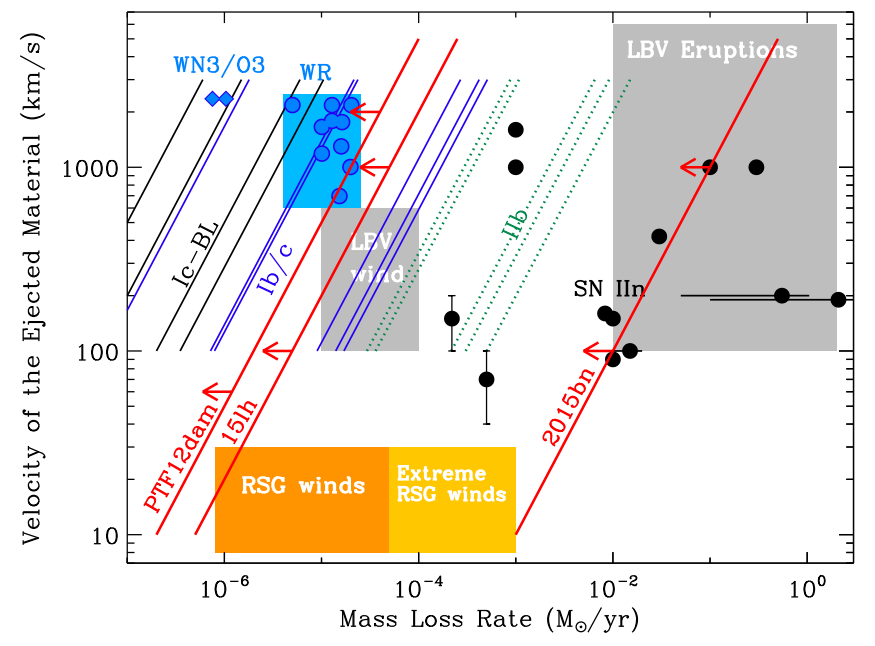

Figure 5. Velocity of the ejected material during mass loss vs. pre-explosion mass-loss rate for $\mathrm{H}$-poor core-collapse SNe (diagonal lines) and type-IIn $\mathrm{SNe}$ (black dots). $\mathrm{H}$-poor $\mathrm{SNe}$ are represented with diagonal lines since radio and $\mathrm{X}$-ray observations constrain the density $\rho_{\mathrm{CSM}}$, which is $\propto \dot{M} / v_{w}$. The black, blue, and dotted green lines mark the sample of type Ic-BL, Ib/c, and IIb SNe from Drout et al. (2016). SLSNe-I and the transient ASASSN$15 \mathrm{lh}$ are in red. For SLSNe-I we conservatively plot the constraints for $E_{k}=10^{51} \mathrm{erg}$, which is a lower limit to the total kinetic energy of the blast wave (even in the case of a magnetar central engine). The properties of galactic WR stars are from Crowther (2007), while WN3/O3 stars are from Massey et al. (2015). The locations of red supergiant environments (RSG) are from de Jager et al. (1988), Marshall et al. (2004) and van Loon et al. (2005). The typical locations of luminous blue variable (LBV) winds and eruptions are from Smith (2014) and Smith \& Owocki (2006). The densest environments that characterize LBV eruptions and type-IIn SNe are not consistent with our deepest SLSNe-I limits. Our tightest constraints on PTF 12dam rule out RSG winds and put PTF $12 \mathrm{dam}$ in the same region of the parameter space as H-stripped SNe with broad spectral features (i.e., Ic-BL).

likely to be compact stars surrounded by a low-density medium at $d>10^{16} \mathrm{~cm}$ at the time of stellar death.

\section{Central Engines in SLSNe-I}

\subsection{Constraints on On-axis and Off-axis Collimated and Non- collimated Relativistic Outflows}

The search for off-axis and on-axis relativistic GRB-like jets in SLSNe-I is motivated by two recent observational findings: (i) the association of SN $2011 \mathrm{kl}$ with GRB 111209A. SN $2011 \mathrm{kl}$ bridges the luminosity gap between GRB-SNe and SLSNe-I, and shows similarities to SLSNe-I (Greiner et al. 2015); and (ii) nebular spectroscopy of the SLSN-I 2015bn revealed close similarities to the enginedriven SN 1998bw, associated with GRB 980425, suggesting that the core of engine-driven SNe and SLSNe-I share some key physical properties and structure (Nicholl et al. 2016a).

Early-time X-ray observations of SLSNe-I acquired at $t \lesssim 40$ days generally rule out on-axis collimated ultrarelativistic outflows of the type associated with energetic long GRBs (Figure 7, cloud of filled gray squares). We constrain the presence of off-axis relativistic outflows by generating a grid of off-axis GRB X-ray afterglows with the broadband afterglow numerical code Boxfit v2 (van Eerten et al. 2012). ${ }^{14}$ The observed X-ray emission depends on the

\footnotetext{
${ }^{14}$ http://cosmo.nyu.edu/afterglowlibrary/boxfit2011.html
}

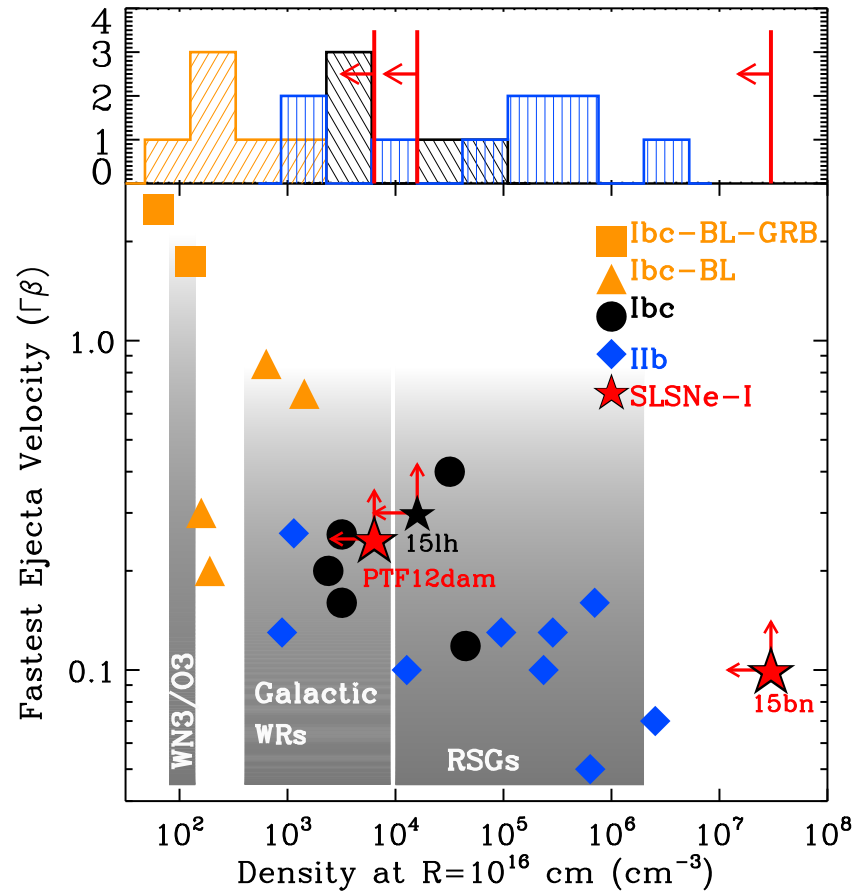

Figure 6. Constraints on the fastest $\mathrm{SN}$ ejecta velocity and environmental density of SLSNe-I with the most sensitive X-ray limits (red stars) and the peculiar transient ASASSN-15lh (black star) in the context of core-collapse stellar explosions from $\mathrm{H}$-stripped progenitors. Gray shaded regions: density in the environments of red supergiant stars (RSGs), Wolf-Rayet stars (WRs), and the recently discovered new type of WR stars, WN3/O3 (de Jager et al. 1988; Marshall et al. 2004; van Loon et al. 2005; Crowther 2007; Massey et al. 2015). Type IIb SNe (blue diamonds) explode in the densest environments, while SNe with broad spectroscopic features (orange squares and triangles) are associated with the lowest-density media. For SLSNe-I we conservatively plot the constraints for $E_{k}=10^{51} \mathrm{erg}$, which is a lower limit to the total kinetic energy of the blast wave (even in the case of a magnetar central engine). In the case of PTF 12dam, our measurements rule out the dense environments associated with RSG winds and are consistent with the clean environments that characterize WRs, WN3/O3, and engine-driven SN explosions. References: van Dyk et al. (1994), Fransson \& Björnsson (1998), Berger et al. (2002), Weiler et al. (2002), Ryder et al. (2004), Soderberg et al. (2005, 2006a, 2006b, 2008, 2010a, 2010b), Chevalier \& Fransson (2006), Roming et al. (2009), Krauss et al. (2012), Milisavljevic et al. (2013), Corsi et al. (2014), Kamble et al. (2014, 2016), Margutti et al. (2014, 2017a), Chakraborti et al. (2015), Drout et al. (2016).

kinetic energy $E_{k}$ of the outflow, the density of the medium $\rho_{\text {CSM }}$ (we explore both an ISM-like medium $n_{\text {CSM }}=$ const and a wind-like medium with $\left.\rho_{\mathrm{CSM}} \propto R^{-2}\right)$, the microphysical shock parameters $\epsilon_{B}$ and $\epsilon_{e}$ (post-shock energy fraction in magnetic field and electrons, respectively), the jet opening angle $\theta_{j}$ and the angle of the jet with respect to the line of sight $\theta_{\text {obs }}$. We explore the predicted X-ray signatures of collimated $\theta_{j}=5^{\circ}$ outflows with $\epsilon_{e}=0.1$ and $\epsilon_{B}=0.01$ (as derived from first-principle simulations of relativistic shocks, e.g., Sironi et al. 2015), isotropic kinetic energy in the range $E_{k, \text { iso }}=10^{52}-10^{55} \mathrm{erg}$, environment density in the range $n=10^{-3}-10 \mathrm{~cm}^{-3}$ (ISM) or mass-loss rate $\dot{M}=10^{-7}-10^{-3} M_{\odot} \mathrm{yr}^{-1}$ (wind) and observed angles $\theta_{\text {obs }} \leqslant 90^{\circ}$. These values are representative of the parameters inferred from accurate modeling of broadband afterglows of GRBs.

Based on these simulations and the X-ray observations from the entire sample of SLSNe-I, we find that relativistic collimated outflows with $E_{k}>10^{51} \mathrm{erg}, n>10^{-3} \mathrm{~cm}^{-3}$ and $\theta_{\text {obs }}<2 \theta_{j}$ are 


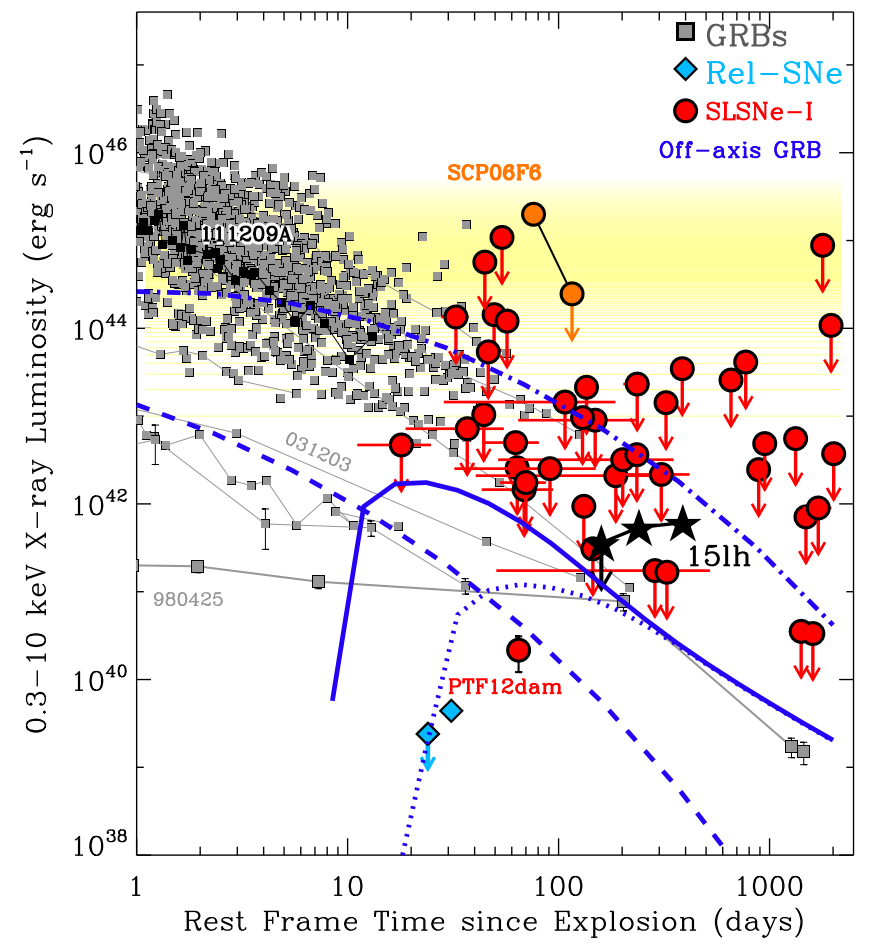

Figure 7. X-ray emission from SLSNe-I (red circles) in the context of detected GRB X-ray afterglows (gray squares, Margutti et al. 2013b), relativistic SNe (blue diamonds, Soderberg et al. 2010b; Margutti et al. 2014) and representative off-axis afterglow models (blue lines) from collimated outflows with $\theta_{\text {jet }}=5^{\circ}, \epsilon_{\mathrm{e}}=0.1, \epsilon_{\mathrm{B}}=0.01, E_{k}=4 \times 10^{52} \mathrm{erg}, \dot{M}=10^{-3} M_{\odot} \mathrm{yr}^{-1}$, $\theta_{\mathrm{obs}}=30^{\circ}$ (thick line) and $\theta_{\mathrm{obs}}=45^{\circ}$ (dotted line). We also show models for $\dot{M}=10^{-7} M_{\odot} \mathrm{yr}^{-1}, \theta_{\text {obs }}=2 \theta_{\text {jet }}, E_{k}=4 \times 10^{52} \mathrm{erg}$ (dotted-dashed line), and $E_{k}=4 \times 10^{50} \mathrm{erg}$ (dashed line). The fast-fading X-ray emission at the location of SCP 06F6 is shown with orange circles (Levan et al. 2013). Black stars: steady X-ray emission at the location of ASASSN-15lh (Margutti et al. 2017b). Black squares: X-ray afterglow of GRB 111209A, associated with the overluminous SN $2011 \mathrm{kl}$ (Greiner et al. 2015). In this plot we show the SwiftXRT limits from the stacked analysis for display purposes. The analysis and results are based on the time-resolved observations. Notably, our deepest limits rule out non-collimated outflows from the weakest GRB explosions, like GRB 980425 (Pian et al. 2000; Kouveliotou et al. 2004).

ruled out. Powerful jets with $E_{k}>10^{52}$ erg expanding in a thick medium with $n \geqslant 10 \mathrm{~cm}^{-3}$ and $\theta_{\text {obs }} \leqslant 30^{\circ}$ are also ruled out. For a wind environment, our observations are not consistent with jets with $E_{k}>10^{50.5} \mathrm{erg}$ expanding in a medium enriched with $\dot{M} \geqslant 10^{-7} M_{\odot} \mathrm{yr}^{-1}$ and $\theta_{\mathrm{obs}}<2 \theta_{j}$. At higher kinetic energies, observations rule out jets with $E_{k}>10^{51.5} \mathrm{erg}, \dot{M} \geqslant 10^{-4} M_{\odot}$ $\mathrm{yr}^{-1}$, and $\theta_{\text {obs }} \leqslant 30^{\circ}$ or $E_{k}>10^{52} \mathrm{erg}, \dot{M} \geqslant 10^{-3} M_{\odot} \mathrm{yr}^{-1}$, and $\theta_{\text {obs }} \leqslant 45^{\circ}$.We are not sensitive to jets viewed at $\theta_{\text {obs }}>30^{\circ}$ for the ISM, and $\theta_{\mathrm{obs}}>45^{\circ}$ for the wind medium.

In the case of PTF 12dam, observations argue against jets with $E_{k}>10^{51}$ erg propagating into a medium with $n>10^{-3} \mathrm{~cm}^{-3}$ or $\dot{M}>10^{-7} M_{\odot} \mathrm{yr}^{-1}$ and $\theta_{\text {obs }}<2 \theta_{j}$. The portion of the parameter space associated with $E_{k}=10^{50.5} \mathrm{erg}, \dot{M} \sim 10^{-6-7} M_{\odot} \mathrm{yr}^{-1}$ and $\theta_{\text {obs }}<2 \theta_{j}$ is also ruled out. Dense environments with $n>$ $10 \mathrm{~cm}^{-3}$ or $\dot{M}>10^{-4} M_{\odot} \mathrm{yr}^{-1}$ would also produce X-ray emission in excess of what we observed for outflows with $E_{k}>10^{51} \mathrm{erg}$ viewed at $\theta_{\mathrm{obs}}<30^{\circ}$.

For the SLSN-I 2015bn observations rule out systems with $E_{k}>10^{52} \mathrm{erg}, n>10^{-3} \mathrm{~cm}^{-3}$, or $\dot{M}>10^{-7} M_{\odot} \mathrm{yr}^{-1}$ for $\theta_{\mathrm{obs}}<2 \theta_{j}$. Even the most energetic outflows in our simulations with $E_{k}>10^{52}$ erg would fall below our detection threshold for $\theta_{\text {obs }}>30^{\circ}$ and the range of densities considered. These observations complement the results from deep radio non-detections of SN 2015bn (Nicholl et al. 2016b), which argue against powerful on-axis or off-axis jets with $E_{k}=2 \times 10^{51} \mathrm{erg}$ propagating into an ISM-like medium with density $n=1 \mathrm{~cm}^{-3}$. $^{15}$

Finally, we consider the observable X-ray signatures of non-collimated mildly relativistic outflows. X-ray observations of the majority of SLSNe-I in our sample are not sensitive to the faint $\mathrm{X}$-ray emission of mildly relativistic non-collimated outflows typical of low-energy GRBs like 980425, 031203, 060218, and 100316D (e.g., Pian et al. 2000; Kouveliotou et al. 2004; Watson et al. 2004; Soderberg et al. 2006a; Margutti et al. 2013a). However, our deepest $\mathrm{X}$-ray limits obtained with the $C X O$ and $X M M$ are sensitive enough to probe the parameter space populated by the weakest GRB-SNe. For the SLSN-I 2015bn, our XMM observations probe and rule out luminosities $L_{x}>2 \times 10^{41} \mathrm{erg} \mathrm{s}^{-1}$ at $t \sim$ 100-300 days, which are comparable to the detected X-ray emission of GRBs 980425 and 031203 at a similar epoch, $L_{x} \sim 10^{41} \mathrm{erg} \mathrm{s}^{-1}$ (Figure 7). Remarkably, in the case of PTF 12dam, CXO observations acquired at the time of optical peak rule out even the faintest non-collimated X-ray emission ever detected from a low-energy GRB (Figure 7), indicating that if PTF 12dam is an engine-driven stellar explosion, the jet never successfully broke out from the stellar envelope, in close analogy to the picture recently suggested for the relativistic SNe 2009bb and 2012ap (Margutti et al. 2014 and references therein).

To conclude, the analysis of our deep X-ray limits in the context of GRB afterglow simulations and the recent finding of similarity in the nebular emission from SN 2015bn with engine-driven $\mathrm{SNe}$, suggest that if SLSNe-I are jet-driven explosions, then either SLSNe-I are powered by very energetic collimated GRB-like outflows that were pointing far away from our line of sight $\left(\theta_{\mathrm{obs}}>30^{\circ}\right)$, or that SLSNeI harbor failed jets that do not successfully break through the stellar envelope and are associated with weak X-ray emission. Late-time radio observations of SN2015bn (Nicholl et al. 2016a) argue against the off-axis relativistic jet scenario in the case of energetic jets (see also Coppejans et al. 2018; Margalit et al. 2018). However, the association of the overluminous SN 2011kl with GRB 111209A suggests that some SLSNe-I might harbor relativistic jets. We therefore propose that, in strict analogy to H-stripped corecollapse SNe of ordinary luminosity (e.g., Mazzali et al. 2008; Xu et al. 2008; Lazzati et al. 2012; Margutti et al. 2014), SLSNe-I are also characterized by a continuum of jet strengths and lifetimes of the central engine.

\subsection{Constraints on Magnetar Central Engines: The Ionization Breakout}

We compute the ionization breakout time and the X-ray luminosity at breakout following Metzger et al. (2014) and Metzger \& Piro (2014). We consider a central engine with an $\mathrm{UV} / \mathrm{X}$-ray luminosity $L$ that releases an energy $L \times t$ in ionizing radiation on a timescale $t$. The radiation ionizes its way through the ejecta on a timescale

\footnotetext{
15 Note that Nicholl et al. (2016b) assumed an ISM-like medium and larger $\epsilon_{B}=0.1$ and $\theta_{j}=10^{\circ}$
} 


$$
t_{\text {ion }} \approx\left\{\begin{array}{l}
120 \text { days } M_{3}^{3 / 4} v_{9}^{-5 / 4} T_{5}^{-0.2}\left(\frac{X_{A}}{0.1}\right)^{1 / 4}\left(\frac{L t}{10^{52} \mathrm{erg}}\right)^{-1 / 4} Z_{8}^{3 / 4},\left(\eta_{\mathrm{thr}} \ll 1\right) \\
110 \text { days } M_{3} v_{9}^{-3 / 2} T_{5}^{-0.4}\left(\frac{X_{A}}{0.1}\right)^{1 / 2}\left(\frac{L t}{10^{52} \mathrm{erg}}\right)^{-1 / 2} Z_{8}^{3 / 2},\left(\eta_{\mathrm{thr}} \gg 1\right),
\end{array}\right.
$$

where $M_{3} \equiv M_{\mathrm{ej}} /\left(3 M_{\odot}\right), T_{5}=T / 10^{5} \mathrm{~K}$ is the temperature of electrons in the recombination layer, $v_{9} \equiv v / 10^{9} \mathrm{~cm} \mathrm{~s}^{-1}, X_{Z}$ is the mass fraction $X_{Z}$ of elements with atomic number $Z=8 Z_{8}$ in the ejecta and

$$
\eta_{\mathrm{thr}} \approx 0.7\left(\frac{L t}{10^{52} \mathrm{erg}}\right)^{-1} M_{3} v_{9}^{-1}\left(\frac{X_{A}}{0.1}\right) T_{5}^{-0.8} Z_{8}^{3}
$$

is the ratio of absorptive and scattering opacity in the ejecta (Metzger et al. 2014). The spin-down timescale $t_{\mathrm{sd}}$ of a magnetar central engine is given by

$$
t_{\mathrm{sd}} \sim 4.7 \mathrm{~d} B_{14}^{-2} P_{\mathrm{ms}}^{2}
$$

and the spin-down luminosity is given by

$$
\begin{aligned}
L_{\mathrm{sd}} & =5 \times 10^{46} B_{14}^{2} P_{\mathrm{ms}}^{-4}\left(1+\frac{t}{t_{\mathrm{sd}}}\right)^{-2} \mathrm{erg} \mathrm{s}^{-1} \\
& \approx 1.1 \times 10^{48} B_{14}^{-2} t_{\mathrm{d}}^{-2} \mathrm{erg} \mathrm{s}^{-1}
\end{aligned}
$$

for $t \gg t_{\mathrm{sd}}, B_{14} \equiv B / 10^{14} \mathrm{G}, P_{\mathrm{ms}} \equiv P / \mathrm{ms}, t_{\mathrm{d}} \equiv t /$ days and we adopted the vacuum dipole spin-down convention employed by Kasen \& Bildsten (2010). For $L=L_{\mathrm{sd}}$ and $t \gg t_{\mathrm{sd}}$ the ionization timescale of Equation (1) can be written as

$$
t_{\text {ion }} \approx\left\{\begin{array}{l}
280 \text { days } M_{3} v_{9}^{-5 / 3} T_{5}^{-4 / 15}\left(\frac{X_{A}}{0.1}\right)^{1 / 3} B_{14}^{2 / 3} Z_{8},\left(\eta_{\text {thr }} \ll 1\right) \\
1273 \text { days } M_{3}^{2} v_{9}^{-3} T_{5}^{-4 / 5}\left(\frac{X_{A}}{0.1}\right) B_{14}^{2} Z_{8}^{3},\left(\eta_{\text {thr }} \gg 1\right) .
\end{array}\right.
$$

The X-ray luminosity at ionization breakout is $L_{x} \approx L_{\mathrm{sd}}\left(t_{\text {ion }}\right) / 14$. In the following we assume that oxygen $\left(Z_{8}=1\right)$ dominates the bound-free opacity in the $\sim \mathrm{keV} \mathrm{X-ray} \mathrm{band,} \mathrm{we} \mathrm{use} \mathrm{and} \mathrm{electron}$ temperature $T=10^{5} \mathrm{~K}, X_{A}=0.1$, velocity of the order of $10^{9} \mathrm{~cm} \mathrm{~s}^{-1}$ and we compute the ionization timescale and the X-ray luminosity at breakout and compare to our X-ray limits and measurements.

For SLSNe-I with well-constrained optical bolometric emission, we use the magnetar parameters $M_{\mathrm{ej}}, P$, and $B$ that best fit the optical bolometric luminosity to estimate $t_{\text {ion }}$ and $L_{x}\left(t_{\text {ion }}\right)$ (Table 4). From Table 4 and Figures 2-4 it is clear that most of the $L_{x}\left(t_{\text {ion }}\right)$ are too faint to be detected and that $t_{\text {ion }}$ is usually much larger than the $\sim 2000$ days that we cover with our observations. However, it is also clear that $t_{\text {ion }}$ and $L_{x}\left(t_{\text {ion }}\right)$ are very sensitive to the magnetar parameters and qualify as excellent probes of central engines in SLSNe-I. Magnetar central engines that would produce very similar optical bolometric outputs that cannot be distinguished with current optical-UV photometry are instead clearly differentiated in their $L_{x}\left(t_{\text {ion }}\right)-t_{\text {ion }}$ properties. As an example, for the bestfitting magnetar parameters of SN 2015bn in Figure $4, L_{x}\left(t_{\text {ion }}\right)$ spans $\sim 5$ orders of magnitude and $t_{\text {ion }}$ ranges from 0.6 to 88 years. For this SLSN-I, our deep X-ray limits obtained with $X M M$ and the combined limit from Swift-XRT favor models with $P>2 \mathrm{~ms}$. The fastest spinning magnetar model with $P=1.5 \mathrm{~ms}$ and relatively small ejecta mass $M_{\mathrm{ej}}=7.4 M_{\odot}$ from Nicholl et al. (2016a) predicts $L_{x}\left(t_{\text {ion }}\right)>10^{43} \mathrm{erg} \mathrm{s}^{-1}$ at $t_{\text {ion }} \sim 0.6$ years and it is therefore disfavored by our X-ray observations (Figure 4).

Figure 8 shows how the X-ray observations from our sample compare to the predictions from the magnetar ionization breakout model. We investigate a wide range of central engine parameters $P=1-7 \mathrm{~ms}$ and $B_{14}=0.2-10 \mathrm{G}$ for ejecta masses $M_{\mathrm{ej}}=1-20 M_{\odot}$. Current X-ray observations are not sensitive to magnetars with $B_{14} \geqslant 2$ (Figure 8 ). For $B_{14}<2$, observations favor models with larger ejecta mass: for $B_{14}=0.2,0.5$, $1.0 \mathrm{G}$ the allowed parameter space is $M_{\mathrm{ej}}>20,7,3 M_{\odot}$. X-ray observations indicate that if a magnetar central engine powers the emission from SLSNe-I then it has to be either associated with a large magnetic field or with a large explosion ejecta mass. These results are independent from (but in agreement with) the values inferred from the modeling of the optical emission from SLSNe-I (Table 4, Inserra et al. 2013; Nicholl et al. 2013).

We end by noting that our analytic treatment of ionization breakout from the supernova ejecta requires confirmation by a more detailed photoionization calculation in future work, as well as a more accurate model for the spectral energy distribution of the young pulsar wind nebula. Also, by adopting a relatively low mass fraction of $X_{Z} \approx 0.1$ of $\mathrm{CNO}$ elements (which contribute most of the bound-free opacity in the $\mathrm{keV}$ range) we may be underestimating the ionization breakout time and thus overestimating the associated X-ray luminosity if the true mass fraction is higher. On the other hand, asphericity in the ejecta (e.g., along the rotation axis) would reduce the breakout time along directions of lower than average density and introduce a viewing angle dependence to the emission. An extension from an analytical 1D model (used here) to detailed multi-D formulations is indeed necessary to fully characterize the expected X-ray signature from ionization breakout, and possibly solve the current tension between the anticipated versus observed spectral features and their evolution in the magnetar model (e.g., Liu et al. 2017).

\section{Summary and Conclusions}

We present the results from an extensive systematic survey of X-ray emission from 26 hydrogen-stripped SLSNe in the local universe with Swift, Chandra, and XMM. These data cover the SLSNe-I evolution from $\sim$ days until 2000 days (restframe) since explosion, reaching $L_{x} \sim 10^{40} \mathrm{erg} \mathrm{s}^{-1}$. The unprecedented depth of these observations provided the deepest limits on the X-ray emission from SLSNe-I to date and enabled the detection of X-ray emission at the location of the slowly evolving SLSN-I PTF 12dam. The major results from our investigation can be summarized as follows:

1. Superluminous X-ray emission $L_{x} \sim 10^{45} \mathrm{erg} \mathrm{s}^{-1}$ of the kind detected at the location of SCP $06 \mathrm{~F} 6$ is not a 


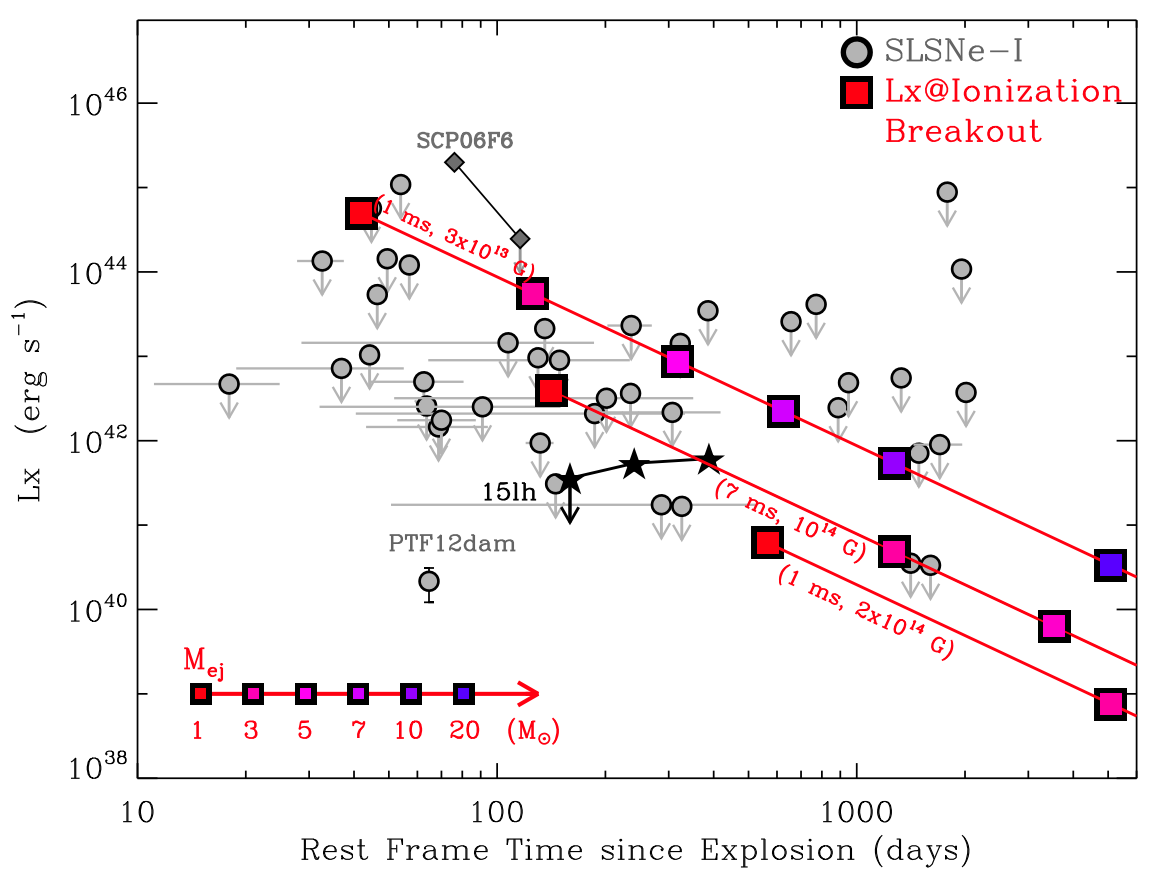

Figure 8. X-ray luminosity at the time of ionization breakout (thick red line) for a variety of representative magnetar parameters, $P=1 \mathrm{~ms}, B=3 \times 10^{13} \mathrm{G}$ (upper line), $P=7 \mathrm{~ms}, B=10^{14} \mathrm{G}$ (middle line), $P=1 \mathrm{~ms}, B=2 \times 10^{14} \mathrm{G}$ (lower line), and for a range of ejecta mass values $M_{\mathrm{ej}}$ between 1 and $20 M_{\odot}$. These calculations assume an oxygen-dominated ejecta composition $\left(Z_{8}=1\right), v=10^{9} \mathrm{~cm} \mathrm{~s}^{-1}, T=10^{5} \mathrm{~K}, X_{A}=0.1$. Our limits (gray dots) rule out the fastest spinning magnetars with $P \leqslant 7 \mathrm{~ms}, B \leqslant 10^{14} \mathrm{G}$ and small ejecta masses $M_{\mathrm{ej}} \leqslant 5 M_{\odot}$. However, we are not sensitive to magnetars with $B_{14} \geqslant 2 \times 10^{14} \mathrm{G}$. Gray diamonds: X-ray emission at the location of SCP 06F6 (Levan et al. 2013). Black stars: X-ray emission at the location of ASASSN-15lh (Margutti et al. 2017b).

common trait of SLSNe-I. Superluminous X-ray emission requires peculiar physical conditions that are likely not shared by the majority of SLSNe-I. If present, its duration is $\leqslant 2$ months at $t<2000$ days and $\leqslant$ few days at earlier epochs $t<100$ days.

2. We place sensitive limits on the sub-parsec environments of the SLSNe-I with the deepest observations and constrain the pre-explosion mass-loss histories of the stellar progenitors of SLSNe-I (Figures 5-6). The most sensitive X-ray observations in our sample rule out the densest environments typical of LBV eruptions and typeIIn $\mathrm{SNe}$ at distances $d>10^{16} \mathrm{~cm}$. For PTF 12dam, the observations point to a clean environment similar to engine-driven $\mathrm{SNe}$ and argue against the extended dense CSM typical of stellar progenitors like RSG. Observations indicate $\dot{M}<2 \times 10^{-5} M_{\odot} \mathrm{yr}^{-1}$. This result suggests that sustained CSM interaction is unlikely to play a key role in the process that powers the luminous display in some SLSNe-I and that at least some SLSN-I progenitors end their lives as compact stars surrounded by a low-density medium at distances $d>10^{16} \mathrm{~cm}$. Our data do not constrain the properties of the nearby $d<10^{16} \mathrm{~cm}$ environment, sculpted by the mass-loss history of the progenitor star in the last $\sim 10 \mathrm{yr}$ before core-collapse.

3. We do not find compelling observational evidence for relativistic outflows in SLSNe-I. SLSNe-I might either be powered by energetic relativistic GRB-like outflows that we did not detect because they were positioned far away from our line of sight $\left(\theta_{\mathrm{obs}}>30^{\circ}\right)$, or might harbor failed jets that do not successfully pierce through the stellar envelope. Deep X-ray observations of PTF 12dam rule out even the weakest emission from uncollimated GRB outflows (Figure 7), suggesting that if PTF 12dam is a jet- driven explosion, then the jet never successfully broke out from the surface (in close similarity to the relativistic $\mathrm{SNe} 2009 \mathrm{bb}$ and 2012ap). However, the luminous SN 2011kl was found in association with the fully relativistic, fully successful jet of GRB 111209A, suggesting that jet-driven explosions can give rise to $\mathrm{SNe}$ more luminous than the average $\mathrm{H}$-stripped core-collapse $\mathrm{SNe}$. We thus propose that, just like H-stripped corecollapse SN, SLSNe-I might also be characterized by a continuum of jet power and central-engine lifetimes.

4. The X-ray ionization breakout is a very sensitive probe of the properties of a hidden magnetar central engine in SLSNe-I. Magnetar central engines that would produce very similar optical/UV displays are instead clearly differentiated in terms of X-ray luminosities and timescales of the ionization breakout (Figures 3-4). Current X-ray observations indicate that if a magnetar central engine powers SLSNe-I, then it has to be either associated with a large magnetic field $B_{14}>2 \mathrm{G}$ or large ejecta mass $\left(M_{\mathrm{ej}}>20,7,3 M_{\odot}\right.$ for $B_{14}=0.2$, $0.5,1.0 \mathrm{G})$.

This X-ray campaign provided constraints on the sub-parsec environment and properties of central engines in SLSNe-I. To further advance our knowledge and understanding of SLSNe-I it is necessary to systematically explore the region of the parameter space with $L_{x}<10^{41} \mathrm{erg} \mathrm{s}^{-1}$ both at very early $(t<30$ days, rest-frame) and late times $(t>1000$ days, restframe), where the emission from an off-axis relativistic jet, weak uncollimated relativistic outflow or magnetar ionization breakout might be found. This parameter space is almost an entirely uncharted territory of exploration and holds promise for future discoveries. 
We thank the referee for constructive criticism and suggestions that helped improve the quality of the paper and the clarity of presentation of our results. We thank A. Kann, K. Murase, and N. Soker for their comments and detailed reading of the manuscript that we posted on the archive. This research has made use of the XRT Data Analysis Software (XRTDAS) developed under the responsibility of the ASI Science Data Center (ASDC), Italy. We acknowledge the use of public data from the Swift data archive. This work is partially based on data acquired with the Swift GO program 1114109 (PI Margutti). The scientific results reported in this article are partially based on observations made by the Chandra X-ray Observatory under program GO6-17052A (PI Margutti), observation IDs 17879, 17880, 17881, 17882, and IDs 13772, 14444, 14446 for PI Pooley. This work is partially based on observations by XMM-Newton, IDs 0743110301, 0743110701, 0770380201, 0770380401 (PI Margutti, proposal 74311). D.C. and R.M. acknowledge partial support from programs No. NNX16AT51G and NNX16AT81G provided by NASA through Swift Guest Investigator Programs. C.G. acknowledges University of Ferrara for use of the local HPC facility co-funded by the "Large-Scale Facilities 2010" project (grant 7746/2011). Development of the Boxfit code was supported in part by NASA through grant NNX10AF62G issued through the Astrophysics Theory Program and by the NSF through grant AST-1009863. Simulations for BOXFIT version 2 have been carried out in part on the computing facilities of the Computational Center for Particle and Astrophysics (C2PAP) of the research cooperation "Excellence Cluster Universe" in Garching, Germany. G.M. acknowledges the financial support from the UnivEarthS Labex program of Sorbonne Paris Cité (ANR10LABX0023 and ANR11IDEX000502).

\section{Appendix X-Ray Observations of SLSNe-I}

Here we provide the details of the X-ray observations of SLSNe-I in the "bronze" and "iron" samples. Table 5 reports the measured fluxes for the entire sample of 26 SLSNe-I analyzed in this paper. For the non-detections we assume a non-thermal power-law spectrum with a photon index $\Gamma=2$ and Galactic absorption.

\section{A.1. SN 2009jh/PTF 09cwl}

Swift-XRT observed SN 2009jh (Quimby et al. 2011c) on 2009 August 29 until 2016 September $25(\delta t=48-1961$ days rest-frame since explosion). No X-ray source is detected at the location of the supernova. With respect to Levan et al. (2013) we add the late-time data set acquired in 2016.

\section{A.2. PTF 09atu}

Swift-XRT observed PTF 09atu (Quimby et al. 2011c) on 2009 August 19 until 2016 October $6(\delta t=49-1785$ days restframe since explosion). No X-ray source is detected at the location of the supernova. With respect to Levan et al. (2013) we add the late-time data set acquired in 2016.

\section{A.3. PTF 09cnd}

Swift-XRT started observing PTF 09cnd (Quimby et al. 2011c) on 2014 August 8 until October 3 ( $\delta t=1487-1531$
Table 5

X-Ray Observations of SLSNe-I

\begin{tabular}{lcccc}
\hline \hline SN & & \multicolumn{3}{c}{$\begin{array}{c}\text { Unabsorbed Flux } \\
(0.3-10 \mathrm{keV})\end{array}$} \\
& $\begin{array}{c}t_{\text {START }} \\
\text { (MJD) }\end{array}$ & $\begin{array}{c}t_{\text {STOP }} \\
(\mathrm{MJD})\end{array}$ & $\begin{array}{c}\text { Instrument } \\
\left(10^{-14} \mathrm{erg} \mathrm{s}^{-1} \mathrm{~cm}^{-2}\right)\end{array}$ & \\
\hline SCP 06F6 & 53949 & 53949 & 13. & XMM \\
& 54043 & 54043 & $<1.40$ & Chandra \\
PTF 09atu & 55062.188 & 55062.328 & $<14.50$ & Swift-XRT \\
& 57667.055 & 57667.055 & $<89.37$ & Swift-XRT \\
PTF 09cnd & 55061.883 & 55062.000 & $<12.02$ & Swift-XRT \\
& 55065.828 & 55065.945 & $<12.35$ & Swift-XRT \\
& 55069.016 & 55069.289 & $<14.47$ & Swift-XRT \\
& 55073.637 & 55073.777 & $<12.43$ & Swift-XRT \\
& 55077.312 & 55077.594 & $<17.12$ & Swift-XRT \\
& 55084.012 & 55084.887 & $<13.73$ & Swift-XRT \\
& 55097.812 & 55097.945 & $<25.94$ & Swift-XRT \\
& 55107.375 & 55107.453 & $<22.40$ & Swift-XRT \\
& 57422.016 & 57422.766 & $<57.30$ & Swift-XRT \\
\hline
\end{tabular}

Note.

${ }^{\text {a }}$ From Levan et al. (2013).

(This table is available in its entirety in machine-readable form.)

days rest-frame since explosion, exposure time of $26 \mathrm{ks}$ ). This data set has been partially presented by Levan et al. (2013). The location of PTF 09cnd was serendipitously observed by Swift between 2016 February 4 and September 23 ( $\delta t=1921-2104$ days rest-frame since explosion, exposure time of $62 \mathrm{ks}$ ). No $\mathrm{X}$-ray source is detected at the location of the supernova. XMM observed the location of PTF 09cnd on 2014 August 8 $(\delta t=1487$ days rest-frame since explosion). The net exposure time is $27.7 \mathrm{ks}$ (EPIC-pn data). No source is detected and we derive a $3 \sigma$ count-rate upper limit of $1.5 \times 10^{-3} \mathrm{c} \mathrm{s}^{-1}$ (0.3-10 keV), which corresponds to an absorbed (unabsorbed) flux $<3.2 \times 10^{-15} \mathrm{erg} \mathrm{s}^{-1} \mathrm{~cm}^{-2}\left(<3.4 \times 10^{-15} \mathrm{erg} \mathrm{s}^{-1} \mathrm{~cm}^{-2}\right)$.

\section{A.4. PTF 10aagc}

Swift-XRT observed PTF 10aagc (Yan et al. 2015) on 2010 November 08 ( $\delta t=79$ days rest-frame since explosion). No $\mathrm{X}$-ray source is detected at the location of the supernova.

\section{A.5. SN 2010md/PTF 10hgi}

Swift-XRT started observing PTF 10hgi (Inserra et al. 2013) on 2010 July 13 until 2010 July 18 ( $\delta t=61-66$ days restframe since explosion). No X-ray source is detected at the location of the supernova as reported by Levan et al. (2013).

\section{A.6. SN 2010gx/CSS100313/PTF 10cwr}

Swift-XRT started observing SN 2010gx (Pastorello et al. 2010; Quimby et al. 2011c; Chen et al. 2013; Inserra et al. 2013; Perley et al. 2016) on 2010 March 19 until 2012 May 14 ( $\delta t=19-659$ days rest-frame since explosion). A portion of this data set has been presented by Levan et al. (2013). Here, we add the observations acquired in 2012. No $\mathrm{X}$-ray source is detected at the location of the supernova.

\section{A.7. SN 2010kd}

Swift-XRT started observing SN 2010kd (Vinko et al. 2010, 2012) on 2010 November 30 until 2016 June 21 $(\delta t=120-1964$ days rest-frame since explosion). With respect 
to Levan et al. (2013), here we include late-time data acquired in 2014 and 2016. No X-ray source is detected at the location of the supernova.

\section{A.8. SN $2011 \mathrm{ke} / \mathrm{CSS} 110406 / \mathrm{PTF} 11 \mathrm{dij}$}

Swift-XRT started observing SN 2011ke (Quimby et al. 2011b; Drake et al. 2013; Perley et al. 2016) on 2011 May 14 until 2012 April $12(\delta t=40-332$ days rest-frame since explosion), as reported by Levan et al. (2013). No X-ray source is detected at the location of the supernova.

The $C X O$ serendipitously imaged the sky location of SN 2011ke on 2015 August $28(\delta t=1411$ days rest-frame since explosion, exposure time of $56 \mathrm{ks}$ ) and on 2016 April 4 $(\delta t=1604$ days rest-frame since explosion, exposure time of $59 \mathrm{ks})$. These data are presented here for the first time. No $\mathrm{X}$-ray source is detected at the location of SN 2011ke and we infer a $3 \sigma$ count-rate upper limit $<5.4 \times 10^{-5} \mathrm{c} \mathrm{s}^{-1}$ and $<5.1 \times 10^{-5} \mathrm{c} \mathrm{s}^{-1}$ in the $0.5-8 \mathrm{keV}$ energy band, for the first and second epoch, respectively. For a non-thermal power-law spectrum with index $\Gamma=2$ these results translate into unabsorbed $0.3-10 \mathrm{keV}$ flux limits of $<6.4 \times 10^{-16}$ and $<6.0 \times 10^{-16} \mathrm{erg} \mathrm{s}^{-1} \mathrm{~cm}^{-2}$.

\section{A.9. PS1-11bdn}

Swift-XRT started observing PS1-11bdn (Lunnan et al. 2014, 2015; Schulze et al. 2018) on 2012 January 11 until 2012 January 28 ( $\delta t=28-35$ days rest-frame since explosion). No $\mathrm{X}$-ray source is detected at the location of the supernova.

\section{A.10. PTF $11 \mathrm{rks} / \mathrm{SN} 2011 \mathrm{~kg}$}

Swift-XRT started observing PTF 11rks (Quimby et al. 2011a; Inserra et al. 2013; Perley et al. 2016) on 2011 December 30 until 2012 January $15(\delta t=11-25$ days restframe since explosion), as reported by Levan et al. (2013). No $\mathrm{X}$-ray source is detected at the location of the supernova.

\section{A.11. SN 2012il/PS1-12fo/CSS120121}

Swift-XRT started observing SN 2012il (Drake et al. 2012; Smartt et al. 2012; Inserra et al. 2013; Lunnan et al. 2014) on 2012 February 13 until 2016 June 25 ( $\delta t=44-1400$ days restframe since explosion). No X-ray source is detected at the location of the supernova. With respect to Levan et al. (2013) we add the 2016 data set.

\section{A.12. DES15C3hav}

Swift-XRT started observing DES15C3hav (Challis et al. 2016) on 2016 June 12 until 2016 September 13 ( $\delta t=202-269$ days rest-frame since explosion). No X-ray source is detected at the location of the supernova.

\section{A.13. iPTF 13ehe}

Swift-XRT observed iPTF 13ehe (Wang et al. 2016a; Yan et al. 2015) on 2014 December $23(\delta t=385$ days rest-frame since explosion). No X-ray source is detected at the location of the supernova.

\section{A.14. $L S Q 14 a n$}

Swift-XRT started observing LSQ 14an (Leget et al. 2014; Jerkstrand et al. 2017; Inserra et al. 2017) on 2014 March 24 until 2014 December 8, with a final observation taken on 2016 August 8 ( $\delta t=196-949$ days rest-frame since explosion). A portion of the data set was presented in Inserra et al. (2017). Here, we present the complete data set of X-ray observations available on LSQ 14an.

\section{A.15. $L S Q 14 f x j$}

Swift-XRT started observing LSQ 14fxj (Smith et al. 2014; Schulze et al. 2018) on 2014 October 29 until 2015 June 16 $(\delta t=64-234$ days rest-frame since explosion). No X-ray source is detected at the location of the supernova.

\section{A.16. $L S Q 14 m o$}

Swift-XRT started observing LSQ 14mo (Leloudas et al. 2015a; Chen et al. 2017) on 2014 January 31, with a last observation taken on 2016 July 24 ( $\delta t=52-774$ days restframe since explosion). No X-ray source is detected at the location of the supernova.

\section{A.17. CSS140925-005854}

Swift-XRT started observing CSS140925-005854 (Campbell et al. 2014; Schulze et al. 2018) on 2014 October 11 until 2015 May $29(\delta t=29-186$ days rest-frame since explosion). No $\mathrm{X}$-ray source is detected at the location of the supernova.

\section{A.18. DES15S2nr}

Swift-XRT started observing DES15S2nr (D'Andrea et al. 2015) on 2015 September 25 until 2016 February 15, with another observation acquired on 2016 September $14(\delta t=32-323$ days res-frame since explosion). No X-ray source is detected at the location of the supernova.

\section{A.19. OGLE15qz}

Swift-XRT observed OGLE15qz (Kangas et al. 2015; Kostrzewa-Rutkowska et al. 2015) on 2015 November 25 ( $\delta t=54$ days rest-frame since explosion). No X-ray source is detected at the location of the supernova.

\section{A.20. OGLE15sd}

Swift-XRT started observing OGLE15sd (Baumont et al. 2015) on 2015 December 8 until 2015 December $9(\delta t=34-212$ days rest-frame since explosion). No X-ray source is detected at the location of the supernova.

\section{A.21. PS16aqv}

Swift-XRT started observing PS16aqv (Chornock et al. 2016) on 2016 March 9 until 2016 June $10(\delta t=53-131$ days rest-frame since explosion). No X-ray source is detected at the location of the supernova.

\section{A.22. PS16op}

Swift-XRT observed PS16op (Dimitriadis et al. 2016) on 2016 January 20 ( $\delta t=57$ days rest-frame since explosion). No $\mathrm{X}$-ray source is detected at the location of the supernova. 


\section{ORCID iDs}

R. Margutti (i) https://orcid.org/0000-0003-4768-7586

B. D. Metzger (1) https://orcid.org/0000-0002-4670-7509

C. Guidorzi (10) https://orcid.org/0000-0001-6869-0835

G. Migliori (1) https://orcid.org/0000-0003-0216-8053

D. Milisavljevic (1) https://orcid.org/0000-0002-0763-3885

E. Berger (i) https://orcid.org/0000-0002-9392-9681

M. Nicholl (1) https://orcid.org/0000-0002-2555-3192

R. Lunnan 난 https://orcid.org/0000-0001-9454-4639

A. Kamble (i) https://orcid.org/0000-0003-0861-5168

M. Modjaz iㅏ https://orcid.org/0000-0001-7132-0333

\section{References}

Angus, C. R., Levan, A. J., Perley, D. A., et al. 2016, MNRAS, 458, 84 Barbary, K., Dawson, K. S., Tokita, K., et al. 2009, ApJ, 690, 1358 Baumont, S., Le Guillou, L., Le Breton, R., et al. 2015, ATel, 8369 Benetti, S., Nicholl, M., Cappellaro, E., et al. 2014, MNRAS, 441, 289 Berger, E., Kulkarni, S. R., \& Chevalier, R. A. 2002, ApJL, 577, L5 Bersten, M. C., Benvenuto, O. G., Orellana, M., \& Nomoto, K. 2016, ApJL, 817, L8

Björnsson, C.-I., \& Fransson, C. 2004, ApJ, 605, 823

Campbell, H., Walton, N., Blagorodnova, N., et al. 2014, ATel, 6524 Chakraborti, S., Soderberg, A., Chomiuk, L., et al. 2015, ApJ, 805, 187

Challis, P., Kirshner, R., Mandel, K., et al. 2016, ATel, 8952

Chatzopoulos, E., Wheeler, J. C., Vinko, J., et al. 2016, ApJ, 828, 94

Chen, T.-W., Nicholl, M., Smartt, S. J., et al. 2017, A\&A, 602, A9

Chen, T.-W., Smartt, S. J., Bresolin, F., et al. 2013, ApJL, 763, L28

Chen, T.-W., Smartt, S. J., Jerkstrand, A., et al. 2015, MNRAS, 452, 1567

Chevalier, R. A., \& Fransson, C. 2006, ApJ, 651, 381

Chevalier, R. A., \& Irwin, C. M. 2011, ApJL, 729, L6

Chomiuk, L., Chornock, R., Soderberg, A. M., et al. 2011, ApJ, 743, 114

Chornock, R., Bhirombhakdi, K., Katebi, R., et al. 2016, ATel, 8790

Coppejans, D. L., Margutti, R., Guidorzi, C., et al. 2018, ApJ, 856, 56

Corsi, A., Ofek, E. O., Gal-Yam, A., et al. 2014, ApJ, 782, 42

Crowther, P. A. 2007, ARA\&A, 45, 177

Dai, L., McKinney, J. C., \& Miller, M. C. 2015, ApJL, 812, L39

Dai, Z. G., Wang, S. Q., Wang, J. S., Wang, L. J., \& Yu, Y. W. 2016, ApJ, 817,132

D'Andrea, C., Smith, M., Sullivan, M., et al. 2015, ATel, 8092

de Jager, C., Nieuwenhuijzen, H., \& van der Hucht, K. A. 1988, A\&AS, 72,259

Dimitriadis, G., Firth, R., Frohmaier, C., et al. 2016, ATel, 8555

Dong, S., Shappee, B. J., Prieto, J. L., et al. 2016, Sci, 351, 257

Drake, A. J., Djorgovski, S. G., Mahabal, A. A., et al. 2012, ATel, 3873

Drake, A. J., Djorgovski, S. G., Mahabal, A. A., et al. 2013, CBET, 3460, 1

Drout, M. R., Milisavljevic, D., Parrent, J., et al. 2016, ApJ, 821, 57

Fransson, C., \& Björnsson, C.-I. 1998, ApJ, 509, 861

Gal-Yam, A. 2012, Sci, 337, 927

Gal-Yam, A., Mazzali, P., Ofek, E. O., et al. 2009, Natur, 462, 624

Gänsicke, B. T., Levan, A. J., Marsh, T. R., \& Wheatley, P. J. 2009, ApJL, 697, L129

Godoy-Rivera, D., Stanek, K. Z., Kochanek, C. S., et al. 2017, MNRAS, 466, 1428

Greiner, J., Mazzali, P. A., Kann, D. A., et al. 2015, Natur, 523, 189

Inserra, C., Nicholl, M., Chen, T.-W., et al. 2017, MNRAS, 468, 4642

Inserra, C., Smartt, S. J., Jerkstrand, A., et al. 2013, ApJ, 770, 128

Jerkstrand, A., Smartt, S. J., Inserra, C., et al. 2017, ApJ, 835, 13

Kalberla, P. M. W., Burton, W. B., Hartmann, D., et al. 2005, A\&A, 440, 775

Kamble, A., Margutti, R., Soderberg, A. M., et al. 2016, ApJ, 818, 111

Kamble, A., Soderberg, A. M., Chomiuk, L., et al. 2014, ApJ, 797, 2

Kangas, T., Dennefeld, M., Harmanen, J., et al. 2015, ATel, 8296

Kann, D. A., Schady, P., Olivares, E. F., et al. 2016, arXiv:1606.06791

Kasen, D., \& Bildsten, L. 2010, ApJ, 717, 245

Kostrzewa-Rutkowska, Z., Wyrzykowski, L., Kozlowski, S., et al. 2015, ATel, 8314

Kouveliotou, C., Woosley, S. E., Patel, S. K., et al. 2004, ApJ, 608, 872

Kozyreva, A., Hirschi, R., Blinnikov, S., \& den Hartogh, J. 2016, MNRAS, 459, L21

Krauss, M. I., Soderberg, A. M., Chomiuk, L., et al. 2012, ApJL, 750, L40
Lazzati, D., Morsony, B. J., Blackwell, C. H., \& Begelman, M. C. 2012, ApJ, 750, 68

Leget, P.-F., Guillou, L. L., Fleury, M., et al. 2014, ATel, 5718

Leloudas, G., Fraser, M., Stone, N. C., et al. 2016, NatAs, 1, 2, [Corrigendum: NatAs, 2016, 1, 34]

Leloudas, G., Patat, F., Maund, J. R., et al. 2015a, ApJL, 815, L10

Leloudas, G., Schulze, S., Krühler, T., et al. 2015b, MNRAS, 449, 917

Levan, A. J., Read, A. M., Metzger, B. D., Wheatley, P. J., \& Tanvir, N. R. 2013, ApJ, 771, 136

Liu, Y.-Q., Modjaz, M., \& Bianco, F. B. 2017, ApJ, 845, 85

Lunnan, R., Chornock, R., Berger, E., et al. 2014, ApJ, 787, 138

Lunnan, R., Chornock, R., Berger, E., et al. 2015, ApJ, 804, 90

Lunnan, R., Chornock, R., Berger, E., et al. 2016, ApJ, 831, 144

Margalit, B., Metzger, B. D., Thompson, T. A., Nicholl, M., \& Sukhbold, T. 2018, MNRAS, 475, 2659

Margutti, R., Kamble, A., Milisavljevic, D., et al. 2017a, ApJ, 835, 140

Margutti, R., Metzger, B. D., Chornock, R., et al. 2017b, ApJ, 836, 25

Margutti, R., Milisavljevic, D., Soderberg, A. M., et al. 2014, ApJ, 797, 107

Margutti, R., Soderberg, A. M., Chomiuk, L., et al. 2012, ApJ, 751, 134

Margutti, R., Soderberg, A. M., Wieringa, M. H., et al. 2013a, ApJ, 778, 18

Margutti, R., Zaninoni, E., Bernardini, M. G., et al. 2013b, MNRAS, 428, 729

Marshall, J. R., van Loon, J. T., Matsuura, M., et al. 2004, MNRAS, 355, 1348

Massey, P., Neugent, K. F., \& Morrell, N. 2015, ApJ, 807, 81

Mazzali, P. A., Valenti, S., Della Valle, M., et al. 2008, Sci, 321, 1185

Metzger, B. D., Margalit, B., Kasen, D., \& Quataert, E. 2015, MNRAS, 454,3311

Metzger, B. D., \& Piro, A. L. 2014, MNRAS, 439, 3916

Metzger, B. D., Vurm, I., Hascoët, R., \& Beloborodov, A. M. 2014, MNRAS, 437, 703

Milisavljevic, D., Soderberg, A. M., Margutti, R., et al. 2013, ApJL, 770, L38

Mineo, S., Gilfanov, M., \& Sunyaev, R. 2012, MNRAS, 426, 1870

Nicholl, M., Berger, E., Margutti, R., et al. 2016a, ApJL, 828, L18

Nicholl, M., Berger, E., Margutti, R., et al. 2017, ApJL, 835, L8

Nicholl, M., Berger, E., Smartt, S. J., et al. 2016b, ApJ, 826, 39

Nicholl, M., Smartt, S. J., Jerkstrand, A., et al. 2013, Natur, 502, 346

Nicholl, M., Smartt, S. J., Jerkstrand, A., et al. 2014, MNRAS, 444, 2096

Ofek, E. O., Cameron, P. B., Kasliwal, M. M., et al. 2007, ApJL, 659, L13

Ofek, E. O., Zoglauer, A., Boggs, S. E., et al. 2014, ApJ, 781, 42

Pastorello, A., Smartt, S. J., Botticella, M. T., et al. 2010, ApJL, 724, L16

Perley, D. A., Quimby, R. M., Yan, L., et al. 2016, ApJ, 830, 13

Pian, E., Amati, L., Antonelli, L. A., et al. 2000, ApJ, 536, 778

Quimby, R. M., Gal-Yam, A., Arcavi, I., et al. 2011a, ATel, 3841

Quimby, R. M., Kulkarni, S. R., Kasliwal, M. M., et al. 2011c, Natur, 474, 487

Quimby, R. M., Sternberg, A., \& Matheson, T. 2011b, ATel, 3344

Romano, P., Guidorzi, C., Segreto, A., Ducci, L., \& Vercellone, S. 2014, A\&A, 572, A97

Roming, P. W. A., Pritchard, T. A., Brown, P. J., et al. 2009, ApJL, 704, L118

Ryder, S. D., Sadler, E. M., Subrahmanyan, R., et al. 2004, MNRAS, 349, 1093

Schulze, S., Krühler, T., Leloudas, G., et al. 2018, MNRAS, 473, 1258

Sironi, L., Keshet, U., \& Lemoine, M. 2015, SSRv, 191, 519

Smartt, S. J., Wright, D., Valenti, S., et al. 2012, ATel, 3918

Smith, M., Firth, R., Dimitriadis, G., et al. 2014, ATel, 6739

Smith, N. 2014, ARA\&A, 52, 487

Smith, N., \& McCray, R. 2007, ApJL, 671, L17

Smith, N., \& Owocki, S. P. 2006, ApJL, 645, L45

Soderberg, A. M., Berger, E., Page, K. L., et al. 2008, Natur, 453, 469

Soderberg, A. M., Brunthaler, A., Nakar, E., Chevalier, R. A., \& Bietenholz, M. F. 2010a, ApJ, 725, 922

Soderberg, A. M., Chakraborti, S., Pignata, G., et al. 2010b, Natur, 463, 513 Soderberg, A. M., Kulkarni, S. R., Berger, E., et al. 2005, ApJ, 621, 908

Soderberg, A. M., Kulkarni, S. R., Nakar, E., et al. 2006a, Natur, 442, 1014

Soderberg, A. M., Nakar, E., Berger, E., \& Kulkarni, S. R. 2006b, ApJ, 638, 930

Soker, N. 2017, RAA, 17, 113

Sukhbold, T., \& Woosley, S. E. 2016, ApJL, 820, L38

Thöne, C. C., de Ugarte Postigo, A., García-Benito, R., et al. 2015, MNRAS, 451, L65

van Dyk, S. D., Weiler, K. W., Sramek, R. A., Rupen, M. P., \& Panagia, N. 1994, ApJL, 432, L115

van Eerten, H., van der Horst, A., \& MacFadyen, A. 2012, ApJ, 749, 44

van Loon, J. T., Cioni, M.-R. L., Zijlstra, A. A., \& Loup, C. 2005, A\&A, 438, 273

van Putten, M. H. P. M., \& Della Valle, M. 2017, MNRAS, 464, 3219

Vinko, J., Zheng, W., Pandey, S. B., et al. 2012, AAS Meeting, 219, 436 
Vinko, J., Zheng, W., Romadan, A., et al. 2010, CBET, 2556

Vreeswijk, P. M., Leloudas, G., Gal-Yam, A., et al. 2017, ApJ, 835, 58

Wang, S. Q., Liu, L. D., Dai, Z. G., Wang, L. J., \& Wu, X. F. 2016a, ApJ, 828,87

Wang, S. Q., Liu, L. D., Dai, Z. G., Wang, L. J., \& Wu, X. F. 2016b, ApJ, 828,87

Watson, D., Hjorth, J., Levan, A., et al. 2004, ApJL, 605, L101
Weiler, K. W., Panagia, N., Montes, M. J., \& Sramek, R. A. 2002, ARA\&A, 40, 387

Woosley, S. E. 2010, ApJL, 719, L204

Xu, D., Watson, D., Fynbo, J., et al. 2008, in COSPAR Meeting 37, 37th COSPAR Scientific Assembly, 3512

Yan, L., Lunnan, R., Perley, D. A., et al. 2017, ApJ, 848, 6

Yan, L., Quimby, R., Ofek, E., et al. 2015, ApJ, 814, 108 